\title{
Analysis of Lacker's Model with an Ageing Factor for the Control of Ovulation and Polycystic Ovary Syndrome
}

\author{
A. CHÁVEZ-ROSS* \\ Centre for Nonlinear Dynamics and its Applicationst, University College London, Gower Street, London, WCIE 6BT, U.K.
}

(Received 3 May 2000; Revised 15 January 2001)

\begin{abstract}
In the present paper, we develop an extensive theoretical analysis of the deterministic model for the control of ovulation in mammals proposed by Mariana et al., (1994), which is an extension of Lacker's model. Mariana et al. incorporated an age decaying factor in follicle maturity, and kept follicle growth law as Lacker first proposed. However, they produced only some numerical examples simulating the new advantages of their model. As a result of the present analysis, we propose an alternative understanding of folliclegenesis, pre-ovulatory follicle selection in mammals, and polycystic ovary syndrome (PCOS) in women. In particular, a minimum oestradiol threshold level required for initial follicular growth is obtained. Relative values of follicle size and age necessary for its development are also determined. We prove that the model controls pre-ovulatory follicle selection rate at a local level. The model is shown to be globally unstable and fails to regulate the selection process. Finally, a discussion on how these results bring new insight to possible causes for PCOS is given.
\end{abstract}

Keywords: Follicle selection; Ovulation; Polycystic ovary syndrome; Age decay; Stability analysis; Gradient system

\section{INTRODUCTION}

The production and fertilization of mammalian eggs is regulated by different elements of the reproductive system. The complete stock of oocytes in the ovary is formed during early fetal development. Only very few of these eggs will be selected for ovulation during the mammal's reproductive age. The remaining oocytes will end up regressing and disappearing from the ovary.

During the ovarian cycle, a group of follicles, each one of which contains an egg, undergoes terminal development. This last phase of ovarian dynamics corresponds to the estrous cycle and is also referred to

*Present address: Department of Mathematics, University of British Columbia, 1984 Mathematics Road, Vancouver, BC V6T 1Z2, Canada. thttp://www.ucl.ac.uk/CNDA 
as the menstrual cycle in the particular case of humans and old world non-human primates. It is now well known that the control process involved during the estrous/menstrual cycle responsible for the selection of ovulatory follicles involves the endocrine system including the hypothalamus, the pituitary glands and the ovary itself (Hodgen, 1982; Hillier, 1994; Spears et al., 1996). By the first half of the menstrual cycle, the ovulatory follicles are selected. However, it remains unclear exactly when and under which circumstances, this selection process occurs. Furthermore, it is also uncertain how the number of ovulating follicles is precisely regulated each cycle.

This selection mechanism can fail and no follicle be able to release an egg. In the case of humans, instead of having one follicle ovulating every month, a considerable number of follicles are selected but do not complete pre-ovulatory maturation. Subsequently, they never ovulate but rather stay in the ovary for some time in various states of regression leading to a polycystic ovary (PCO). PCO together with its adjacent consequences such as anovulation or evidence of androgen excess, accounts for the polycystic ovary syndrome (PCOS). Although suitable treatment is now available for this common syndrome amongst women of reproductive age, a better understanding on how the selection process fails leading to $\mathrm{PCO}$ is still required.

In the early eighties, Lacker was the first to publish a mathematical model reflecting the dynamics of many growing follicles during the follicular phase of the menstrual cycle (Lacker, 1981). Many assumptions are made about the complex pituitary-ovary system and follicular development in order to obtain a suitable and manageable system of ordinary differential equations. This model is able to reflect the basic features of the follicular phase of the cycle. Such features involve the emergence of pre-ovulatory and ovulatory follicles, control of the selection number, and decay of the remaining non-selected follicles.

Lacker's model also simulates anovulation, which could be interpreted as PCO, either by manipulation of the relevant parameters or by changes on the initial conditions of the system. His model is able to select many pre-ovulatory follicles that never ovulate, but rather remain stuck with a fixed size. However, this model presents some unrealistic features, being one of these, the fact that strictly the largest follicles are always selected for pre-ovulatory maturity. This is a consequence of all follicles growing exactly in the same way.

In the present paper, we study an interesting modification of Lacker's model, developed by Mariana et al. (1994). This modified model avoids the strong hierarchy amongst the growing follicles, and the initial largest ones are not always the selected follicles.

It has been biologically proposed that the selected follicles are amongst the largest ones, yet they are not necessarily the largest (Gougeon and Lefèvre, 1983; Ledger and Baird, 1995). Hence, to consider that the strictly largest follicles are always selected is somehow unrealistic. The maturity of the follicle is determined by both size and oestradiol production and thus, selection seems to depend on the right combination of these two characteristics. Moreover, the way follicles react to hormone stimulation has not yet been well determined. Therefore, although it is possible to assume that the largest one produces the largest amount of oestradiol, it may not be necessarily selected.

Mariana et al.'s model is a way to improve Lacker's model to be dynamically more interesting since the selected follicles are not determined in such an obvious manner; i.e. we do not know which are the follicles to reach pre-ovulatory stage. Mariana and collaborators use the same interaction between follicles suggested by Lacker, but they additionally propose that these follicles intrinsically deteriorate. This is achieved by incorporating another variable that reflects disintegration for each follicle independent of hormone stimulation. This atrophy is referred to as follicle ageing.

The morphological and biochemical pathways through which follicles regress is known as atresia. Atresia is considered an important mechanism involved in follicle development (Faddy and Jones, 1988). Ever since the complete stock is formed before birth, there is a continuous follicular depletion. The initial number of follicles decreases with the age of the mammal. Hence, follicles' fate is to die rather than 
ovulate. The biological nature of the ageing process proposed by Mariana et al., however, is not clearly specified and is somehow arbitrary. Nevertheless, it is a plausible assumption and leads to a realistic model for the selection of the ovulatory follicle.

Contrary to the extensive analysis of Lacker's model available in the literature (Lacker, 1981; Akin and Lacker, 1984; Lacker et al., 1987; Lacker and Akin, 1988; Lacker and Percus, 1991; Chávez-Ross et al., 1997), there is no theoretical analysis for this model. Mariana et al. only presented some numerical examples in order to show that their model is able to reflect the basic features of control of ovulation and its new advantages. Therefore, a thorough theoretical and numerical analysis is developed in this paper, leading to a better understanding of the ageing effects on the cycle dynamics.

The present manuscript is organized as follows. We begin by giving a short physiological description of the ovulation cycle in mammals. Then, in "Lacker's model and the age decaying factor section", we briefly describe both Lacker's and Marians's et al. models. In "The simplified system section", we develop a thorough analysis of a simplified case of the latter one, where we assume all follicles grow with the same initial size and age. In "Dynamics of follicles with different initial sizes and same age section", we continue by studying the situation when follicles grow with different initial size, but same initial age. We then develop a stability analysis for the most general case where all follicles grow with different size and age. In "Further results section", we produce some numerical examples that offer some insight about the global instability of the model in terms of possible causes for PCOS. Finally, we discuss all the new characteristics of the model with the age decaying factor, and what they offer as putative explanations for either infertility or PCOS.

\section{A SHORT BIOLOGICAL DESCRIPTION OF THE MENSTRUAL CYCLE IN PRIMATES}

The follicular phase of the menstrual cycle begins once steroid serum levels are adequate so that the pituitary gland is able to produce Follicular Stimulation Hormone (FSH) and Luteinizing Hormone ( $\mathrm{LH})$. This is in response to the Gonadotropic Releasing Hormone ( $\mathrm{GnRH}$ ), previously released by the hypothalamus. At that particular stage, the group of follicles that reached the Graafian state start secreting oestradiol into the blood stream and continue growing. Such follicular steroidogenesis and growth is signalled by both FSH and LH. When certain serum levels of oestradiol are reached, the pituitary gland stops its FSH production. The follicles that did not reach pre-ovulatory maturity start regressing due to the lack of FSH, which is necessary for their further development. Selected follicles continue growing and monopolize the oestradiol production inside the ovary. In this way, the negative feedback upon the pituitary is maintained and smaller follicles are prevented from reaching pre-ovulatory size and secreting oestradiol.

A few days later, the mid-cycle LH surge takes place stimulating spontaneous ovulation of the preovulatory follicles, a few hours later. Once each follicle has ovulated, it is transformed into the corpus luteum. These corporea lutea carry on with the oestradiol and progesterone production to keep the negative feedback effect upon FSH pituitary secretion. If pregnancy is not initiated, the corporea lutea regress and stop their steroid production and the negative feedback ceases. Hence, FSH and LH levels are restored and the menstrual cycle starts again.

In summary, this is the basic feedback mechanism and follicular development taking place during the menstrual cycle. However, it is worth mentioning that many more paracrine and autocrine interactions occur, which make the cycle the result of a complicated signalling network. We have therefore just described the basic features considered in the mathematical models.

\section{LACKER'S MODEL AND THE AGE DECAYING FACTOR}

From the above description of the menstrual cycle, Lacker considered the gonadotropins (FSH and LH) 
release rate as a function of oestradiol blood concentration. The follicle growth rate is in turn determined by the serum concentration of $\mathrm{LH}$ and FSH. In addition, he made the following assumptions. Follicle size, maturity and oestradiol secretion are all proportional. All follicles respond identically to gonadotropins and obey the same growth law. LH and FSH are considered as the same hormone. The pulsatile effects of GnRH upon pituitary release of gonadotropins are ignored. Hormone clearance and transport to the corresponding target organ are much faster than menstrual cycle. Hence, hormone levels and pituitary response are assumed to be in equilibrium.

After these basic assumptions, Lacker proposed the following equation for the growth rate of each interacting follicle,

$$
\frac{\mathrm{d} x_{i}}{\mathrm{~d} t}=x_{i} g\left(x_{i}, X\right)
$$

where, $x_{i}$ represents each follicle size or oestradiol production. The total oestradiol concentration is given by,

$$
X=\sum_{i=1}^{N} x_{i}
$$

for $N$ growing follicles. The growth function $g$ is the same for each follicle and is given by,

$$
g\left(x_{i}, X\right)=K-D\left(X-M_{1} x_{i}\right)\left(X-M_{2} x_{i}\right)
$$

The feedback loop between the pituitary gland and the ovary is then implicitly established through $X$. This implies that effects of LH and FSH, which Lacker considered as a single hormone, remain implicit in the system. The constant parameter $K$ accounts for the initial follicle exponential growth. $D$ is a threshold parameter to indicate ovulation from anovulation, while $M_{1}$ and $M_{2}$ are involved in hormone sensitivity. For a detailed analysis and study of Lacker's system behavior, please refer to Chávez-Ross et al. (1997). In Fig. 1, we depict an example for Lacker's model where, depending on the initial size distribution, follicles ovulate or get stuck.
In Mariana et al.'s, $y_{i}$ is introduced as the age of the $i$ th follicle. After the change of variables $x_{i}=z_{i} y_{i}$, where $z_{i}$ accounts for the maturity variable used by Lacker, the following system is obtained

$$
\frac{\mathrm{d} z_{i}}{\mathrm{~d} t}=x_{i} g\left(x_{i}, X\right)
$$

and

$$
\frac{\mathrm{d} y_{i}}{\mathrm{~d} t}=-\mu y_{i} .
$$

where $\mu$ is the ageing parameter and the function $g\left(x_{i}\right.$, $X$ ) is given by Eq. (3). Since the ageing dynamics is strictly decreasing, $\mu>0$ and $y_{i}$ should be understood not as a chronological age of the follicle, but rather as a deteriorating capacity. The modified dynamics for the follicle growth proposed by Mariana et al., is then

$$
\frac{\mathrm{d} x_{i}}{\mathrm{~d} t}=y_{i} x_{i} g\left(x_{i}, X\right)-\mu x_{i}, \quad \frac{\mathrm{d} y_{i}}{\mathrm{~d} t}=-\mu y_{i}
$$

For this model, pre-destination for the selection of the largest follicles is no longer valid (Mariana et al., 1994). It is observed that selected follicles are amongst the largest, but they are not necessarily the largest ones (see Fig. 4). Let us proceed with the theoretical analysis of system Eq. (4).

\section{THE SIMPLIFIED SYSTEM}

The first step we choose for analyzing the basic features of this model is to simplify the system by considering a number of follicles with same initial maturity. Let us suppose that from $N$ follicles starting the cycle, $M$ have the same initial maturity $X / M$ and the remaining $N-M$ follicles have zero initial maturity. Suppose, moreover that all follicles have the same initial age $y$. System (Eq. (4)) now reads

$$
\begin{aligned}
& \frac{\mathrm{d} X}{\mathrm{~d} t}=y\left(K X+D \gamma X^{3}\right)-\mu X \\
& \frac{\mathrm{d} y}{\mathrm{~d} t}=-\mu y
\end{aligned}
$$



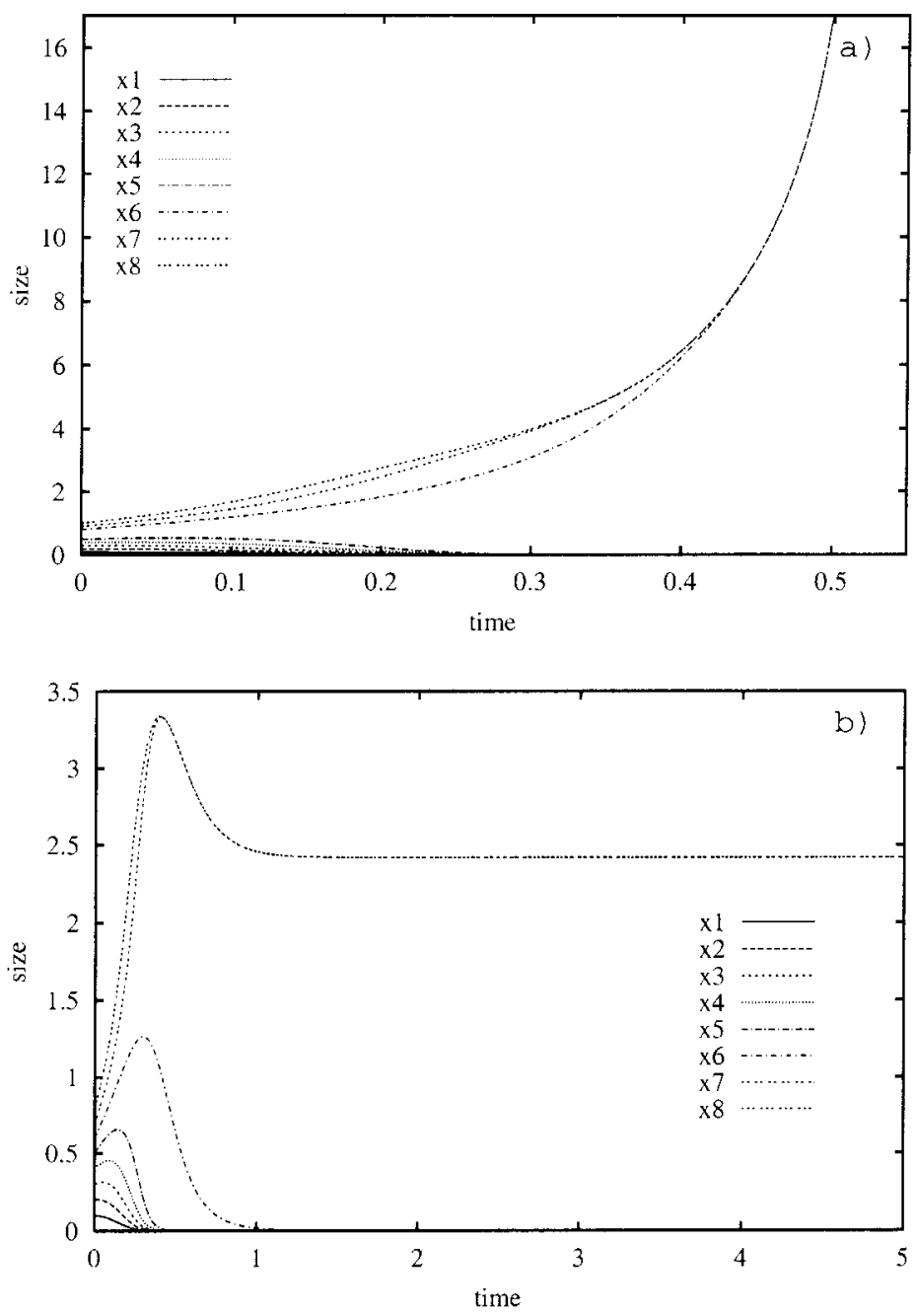

FIGURE 1 A numerical simulation of eight follicles interacting according to Lacker's original model given by (Eqs $(1-3)$ ). A 4th order Runge-Kutta numerical method with a step size of 0.001 was programmed in $\mathrm{C}$ language to simulate the cycle. The parameter values used are $K=5.0, D=0.5, M_{1}=2.9, M_{2}=3.9$. (a) Three follicles $x_{6}, x_{7}$ and $x_{8}$ have similar and relatively large initial sizes with the remaining five follicles $x_{1}, \ldots x_{5}$ having small initial sizes. Follicles $x_{6}, x_{7}$ and $x_{8}$ ovulate in a finite time and the remainder die by atresia. (b) Follicles $x_{7}$ and $x_{8}$ have relatively large initial sizes and the remainder $x_{1}, \ldots, x_{6}$ have smaller initial sizes. The two largest follicles tend to a constant maturity value as $t \rightarrow \infty$ and the rest atrophy by atresia.

where;

$$
\gamma=-\left(1-M_{1} / M\right)\left(1-M_{2} / M\right)
$$

involves the parameters $M_{1}$ and $M_{2}$ of function $g$, as well as the number of identically maturing follicles, $M$. The sign of $\gamma$ prescribes different types of behaviour just as in Lacker's model. $\gamma$ thus determines follicle sensitivity to hormone stimulation, and its sign indicates ovulation or anovulation. It is easy to verify that $(X, y)=(0,0)$ is a local stable equilibrium point for any $\mu>0$. For the particular case of $\mu=0$ system (Eq. (5)) reduces to

$$
\frac{\mathrm{d} X}{\mathrm{~d} t}=y\left(K X+D \gamma X^{3}\right)
$$

where $y$ remains constant for all $t$. If $y=1$, Eq. (7) is the simplified version of Lacker's model whose 


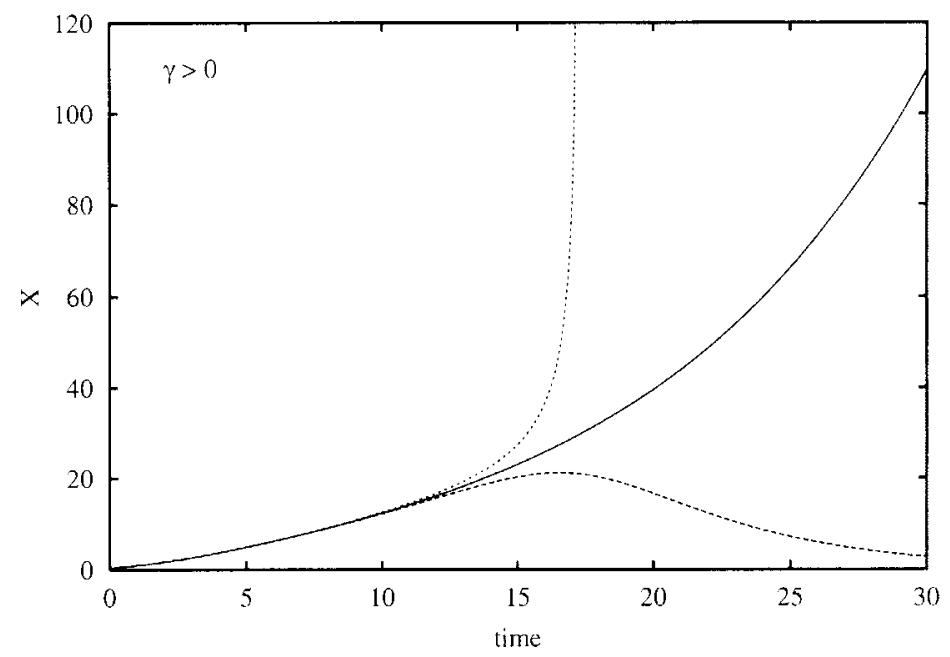

FIGURE 2 Separatrix of the dynamics given by function (Eq. (9)). Here, $y_{0}=1.0, \gamma=0.01>0, \mu=0.2$, so that $X_{0}^{*}=0.477106$. Given $\epsilon=0.001$, for (a) $X_{0_{1}}=X_{0}^{*}+\epsilon$, the solution grows to infinity at a finite time, while for (b) $X_{0_{2}}=X_{0}^{*}-\epsilon$, the solution corresponds to an atretic follicle. The separatrix grows to infinity as $t \rightarrow \infty$.

behavior is carefully analyzed in (Chávez-Ross et al., 1997).

By solving Eq. (5b) and substituting into Eq. (5a), we obtain the non-autonomous differential equation

$$
\frac{\mathrm{d} X}{\mathrm{~d} t}=y_{0} \mathrm{e}^{-\mu t}\left(K X+D \gamma X^{3}\right)-\mu X,
$$

where $y_{0}=y(0)$. The only possible equilibrium point is $X=0$, which is locally stable. After solving this equation, we obtain the following solution

$$
\begin{aligned}
X(t)= & {\left[-\frac{D \gamma}{2 K}\left(2+2 \nu \mathrm{e}^{\mu t}+\nu^{2} \mathrm{e}^{2 \mu t}\right)\right.} \\
& \left.+k \exp \left(\frac{2 \mathrm{e}^{-\mu t}}{\nu}+2 \mu t\right)\right]^{-1 / 2}
\end{aligned}
$$

where,

$$
\begin{gathered}
k=\left[\frac{1}{X_{0}^{2}}+\frac{D \gamma}{2 K}\left(2+2 \nu+\nu^{2}\right)\right] \exp \left(\frac{-2}{\nu}\right), \\
\nu=\mu /\left(K y_{0}\right) \text { and } X_{0}=X(0)>0
\end{gathered}
$$

whenever $\gamma>0$, there exists a separatrix of the dynamics with initial condition

$$
X_{0}^{*}=\left[\frac{K / D \gamma}{1 / 2\left(\mathrm{e}^{2 / \nu}-1\right) \nu^{2}-\nu-1}\right]^{1 / 2} .
$$

Therefore, if (a) $X_{0}>X_{0}^{*}$, solution (9) grows to infinity in finite time, whereas if (b) $0<X_{0}<$ $X_{0}^{*}, X(t)$ tends to zero as time tends to infinity (see Fig. 2). The separatrix grows to infinity in indefinite time or it reaches its maximum in infinite time. It is important to notice that for Lacker's model, where $\mu=0$ and $y=1$, the separatrix does not exist, and all follicle dynamics tends to infinity (Chávez-Ross et al., 1997).

To show how the separatrix initiating from $X_{0}^{*}$ is found, let us compute the time derivative of Eq. (9). By writing $X^{\prime}(t)=F\left(t, X_{0}\right)$, from $F\left(t, X_{0}\right)=0$ we obtain $X_{0}=f(t)$, so that $X_{0}^{*}=\lim _{t \rightarrow \infty} f(t)$. Thus, $X_{0}^{*}$ is the initial condition for which the maximum value is attained in infinite time. Moreover, it is possible to prove that $X_{0}^{*}$ exists for any $\mu>0$. Namely, given $\mu>0$, there always exists a value for the initial condition above which all solutions escape to infinity in finite time. Notice that if $\gamma<0, X_{0}^{*}$ is no longer real and hence, there is no separatrix for the anovulation case. On the other hand, when $\gamma>0, X_{0}^{*}$ is inversely proportional to $\gamma$. This means that the initial condition 
for the separatrix decreases as $\gamma$ increases, and vice versa.

Biologically speaking, $X_{0}^{*}$ represents a threshold value for the total follicle size that did not appear in Lacker's model (Chávez-Ross et al., 1997). We notice that when $\mu=0$ and $y=1, X^{\prime}(t)$ does not explicitly depend on $t$, so $X_{0}^{*}$ does not exist for Lacker's model. Hence, if at the beginning of the cycle, the initial sum of follicle sizes do not exceed such a threshold, there is no hope for any follicle to ovulate, and all of them rather atrophy and die. This may imply that this model is in fact reflecting the cycle dynamics even before the follicular phase.

In contrast, for the case when $X(t)$ does not grow to infinity in finite time, it is possible to compute a critical value for $\mu$. This critical $\mu$ determines whether the follicle grows at the beginning of the cycle, or it immediately decreases. From the critical points of Eq. (8), the maximum size of the follicle is reached at

$$
t_{c}=\frac{1}{\mu} \ln \left[\frac{\left(1+D \gamma X^{2}\right)}{\nu}\right] .
$$

Given any $X>0$ (in particular let $X=X_{0}$ ) the maximum of solution $X(t)$ exists as long as $t_{c} \geq 0$. Moreover, given $\gamma$ as in Eq. (6) and $y_{0}>0$, if $\gamma>0$ and $0<X_{0}<X_{0}^{\text {ast }}$, there is a critical value $\mu^{*}=$ $\left(1+D \gamma X_{0}^{2}\right) K y_{0}$, such that if $0<\mu \leq \mu^{*}$, a maximum exists. This means that although the follicle is destined to die since its initial size is smaller than the minimum size required to ovulate: If $\mu \leq \mu^{*}$, it will be able to grow at first, and then decrease (see Fig. 3b). In contrast, if $\mu>\mu^{*}$, follicle atretic parameter is so large for its initial size that it never grows but immediately regresses (see Fig. 3a).

In the case of $\gamma<0$, the follicle, no matter its initial size, will end up atrophying and dying, i.e. $X(t) \rightarrow 0$ as $t \rightarrow \infty$. However, when $\nu<1$ and

$$
X_{0} \leq \sqrt{\frac{\nu-1}{\gamma D}}
$$

a maximum exists since for those conditions, $0<$ $\mu \leq \mu^{*}$, i.e. $t_{c} \geq 0$ as Fig. $3 b$ shows. Otherwise, the solution is strictly decreasing (see Fig. 3a). The maximum is obtained from Eq. (8), when

$$
\begin{gathered}
\mathrm{d} X / \mathrm{d} t=G(t, X)=0 \\
X_{\max }=\sqrt{\frac{\nu \mathrm{e}^{\mu t_{c}}-1}{\gamma D} .}
\end{gathered}
$$

Finally, for $\gamma<0$, if $\nu>1$ and $\mu>\mu^{*}$, the solution is also monotonically decreasing. The critical value $\mu^{*}$ corresponds to an ageing parameter threshold, which determines different types of behavior. Except for the case when $X(t)$ tends to infinity in finite time, i.e. ovulation. Once an initial oestradiol concentration is given, follicles grow at the beginning of the cycle, as long as $0<\mu \leq \mu^{*}$, i.e. as long as its decaying parameter is not too strong for it to die.

To finish this section, if we decrease $\mu$ even further when $\gamma<0$, the follicle appears to get stuck inside the ovary (see Fig. 3c). It can also be noticed that for very small $\mu$, the maximum size approaches the equilibrium value for Lacker's simplified model, i.e.

$$
X_{\max } \rightarrow\left[-\frac{1}{\gamma}\right]^{1 / 2} \quad \text { as } \mu \rightarrow 0
$$

This limit agrees with Lacker's simplified growth Eq. (7) for $y=1$ and $\mu=0$.

The features observed in Fig. $3 b$, where $\gamma<0$, and Fig. $3 c$ represent anovulation where the stuck follicle eventually disappears from the ovary. This is more realistic than the type of anovulation reflected in Lacker's model since the ovary does not hold the stuck pre-ovulatory follicle indefinitely. Instead, it disappears after some time despite the fact that its response to gonadotropins is adequate for initially maintaining a pre-ovulatory size.

We may deduce that ovulation occurs in the same fashion as ovulation in Lacker's model. However, we cannot conclude the same for the anovulation case, where the follicle reaches a non-trivial stable equilibrium. In contrast, for this revised model, zero is the only equilibrium point, meaning that due to its ageing factor, the stuck follicle eventually dies rather 

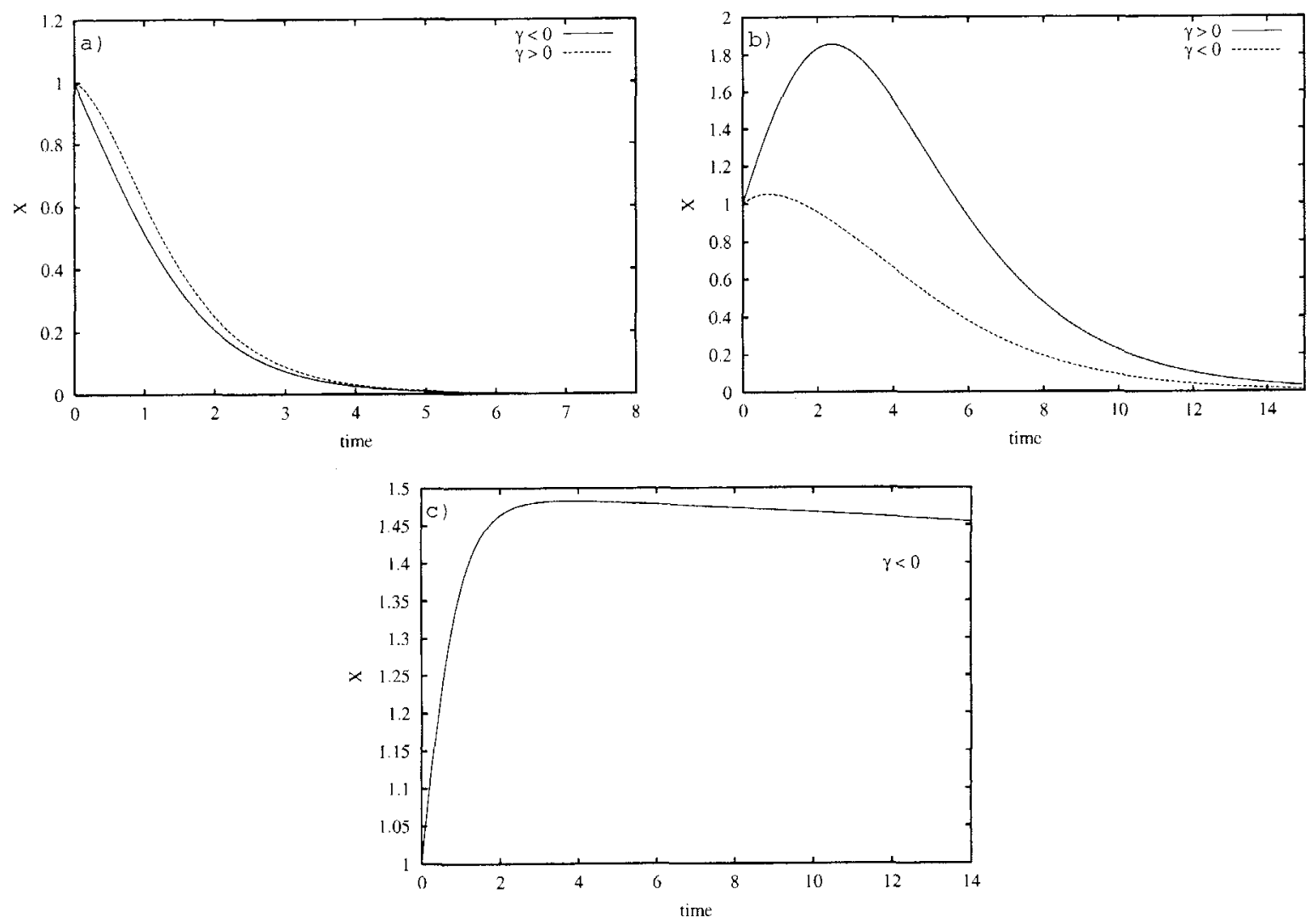

FIGURE 3 Solution (Eq. (9)) for $K=1.0, y_{0}=1.0, X_{0}=1.0, \gamma=0.01>0$, and $\gamma=-0.4275<0$. (a) For $\mu=1.1, \mu>\mu^{*}$, i.e. the solution is monotone decreasing for both $\gamma>0$ and $\gamma<0$. In either case, the follicle is not able to grow at all. (b) For $\mu=0.4$ and $\gamma>0$, $0<\mu<\mu^{*}$, i.e. the solution is unimodal. Whilst for $\gamma=-0.4275<0, X_{0}<1.185$ satisfying inequality (Eq. (11)), i.e. the solution also has a maximum value. (c) Anovulation for $\mu=0.05$ and $\gamma=-0.4275<0$. The follicle appears to tend to the equilibrium value of the simplified Lacker's model, $X_{\max }=1.53$.

than remaining indefinitely at a fixed large size. However, the ambiguity in the time scale of the ageing dynamics complicates a proper biological interpretation of this variable.

\section{ANALYSIS FOR MANY INTERACTING FOLLICLES}

In this section, we study and discuss the dynamics of many interacting follicles. Initially, we consider them to be different in size and same age. Later, we extend our analysis to the case where they differ both in age and size.

\section{Dynamics of Follicles with Different Initial Sizes and Same Age}

For this case, the follicle growth function is given by

$$
g\left(x_{i}, X, y\right)=y g\left(x_{i}, X\right)-\mu
$$

for all follicles. Like the original Lacker's growth rate function, it can be separated using three different functions,

$$
\begin{aligned}
& \frac{\mathrm{d} x_{i}}{\mathrm{~d} t}=x_{i}\left(y \delta(X)\left[\rho(X)+\xi\left(p_{i}\right)\right]-\mu\right) \\
& \frac{\mathrm{d} y}{\mathrm{~d} t}=-\mu y,
\end{aligned}
$$


where the separated functions are the same as in Akin and Lacker (1984), i.e.

$$
\begin{aligned}
& \xi\left(p_{i}\right)=D p_{i}\left(M_{1}+M_{2}-M_{1} M_{2} p_{i}\right), \\
& \rho(X)=\frac{K}{X^{2}}-D, \\
& \delta(X)=X^{2} .
\end{aligned}
$$

By rescaling time

$$
\frac{\mathrm{d} \tau}{\mathrm{d} t}=\delta(X)
$$

the resulting interaction, age and intensity dynamics are

$$
\begin{aligned}
\frac{\mathrm{d} p_{i}}{\mathrm{~d} \tau} & =y p_{i}\left[\xi\left(p_{i}\right)-\bar{\xi}(\bar{p})\right] \\
\frac{\mathrm{d} y}{\mathrm{~d} \tau} & =-\frac{\mu}{\delta(X)} y \\
\frac{\mathrm{d} X}{\mathrm{~d} \tau} & =X\left[y \rho(X)+y \bar{\xi}(\bar{p})-\frac{\mu}{\delta(X)}\right]
\end{aligned}
$$

where, $p_{i}=x_{i} / X$ is the $i$ th follicle relative size.

By further time rescaling, $\mathrm{d} \tau^{\prime} / \mathrm{d} \tau=y$, we get the following interaction dynamics

$$
\frac{\mathrm{d} p_{i}}{\mathrm{~d} \tau^{\prime}}=p_{i}\left[\xi\left(p_{i}\right)-\bar{\xi}(\bar{p})\right],
$$

where $\bar{\xi}(\bar{p})=\sum_{i=1}^{N} p_{i} \xi\left(p_{i}\right)$. Consequently, for Eq. (16), we have the same equilibrium condition as for the corresponding interaction dynamics for Lacker's model, which leads to the same $M$-fold equilibrium point

$$
\bar{p}_{M}=(\underbrace{\frac{1}{M}, \frac{1}{M}, \ldots, \frac{1}{M}}_{M}, \overbrace{0, \ldots, 0}^{N-M})
$$

It is possible to express Eq. (16) as a gradient system on the unit sphere, and then prove that its symmetric equilibrium point (Eq. (17)) is stable as in Lacker and Akin (1988). Thus, if $\bar{\xi}(\bar{p})=\lambda$, we have that
Theorem 5.1. An M-fold non-degenerate equilibrium $y_{e}$ of the gradient system

$$
\frac{\mathrm{d} y}{\mathrm{~d} t}=-\nabla_{s} V
$$

for $y \in S$ is stable if and only if the common value $\lambda>0$, and either $\xi_{i}^{\prime}\left(a_{i}^{2}\right)<0$ for all non-zero coordinates $a_{l}, \ldots, a_{M}$ or $\xi_{i}^{\prime}\left(a_{i}^{2}\right) \geq 0$ for exactly one non-zero coordinate and

$$
\sum_{i=1}^{M} \frac{1}{\xi_{i}^{\prime}\left(a_{i}^{2}\right)}>0
$$

Therefore, there is a stable equilibrium point attracting the $M$ largest follicles, while the remaining $N-M$ smaller ones regress and die by atresia. The $M$-fold equilibrium point (Eq. (17)) outside the unit sphere is determined by the corresponding intensity dynamics given by Eqs. (15b) and (15c).

When substituting back the values of the rescaled function $\delta(X)$ as well as expressing Eqs. (15b) and $(15 c))$ in terms of $t$, these two equations are equivalent to the ones describing the simplified system (Eq. (5)), for which a thorough stability analysis has been already developed in "The simplified system section".

Finally, to prove that ovulation occurs in finite time, we study the asymptotic dynamics in the rescaled time (Eq. (14)). Rescaling Eqs. (15b) and (15c) using $\mathrm{d} \tau^{\top} / \mathrm{d} \tau=y$, we obtain

$$
\begin{aligned}
& \frac{\mathrm{d} y}{\mathrm{~d} \tau^{\prime}}=-\frac{\mu}{\delta(X)} \\
& \frac{\mathrm{d} X}{\mathrm{~d} \tau^{\prime}}=X\left[\rho(X)+\bar{\xi}(\bar{p})-\frac{\mu}{y \delta(X)}\right] .
\end{aligned}
$$

For sufficiently large $X$ and $\bar{p} \approx \bar{p}_{M}$, we have that $\mathrm{d} y / \mathrm{d} \tau^{\prime} \approx 0$. Implying that $y \approx y_{c}$, for $y_{c}$ constant and

$$
\frac{\mathrm{d} X}{\mathrm{~d} \tau^{\prime}}>X(-D+\lambda)>0
$$

for $\lambda=\xi(1 / M)>D$. Since $\delta(X)>0$ and $y>0, \tau(t)$ and $\tau(\tau)$ are both invertible, and since $\lambda>D$, we 

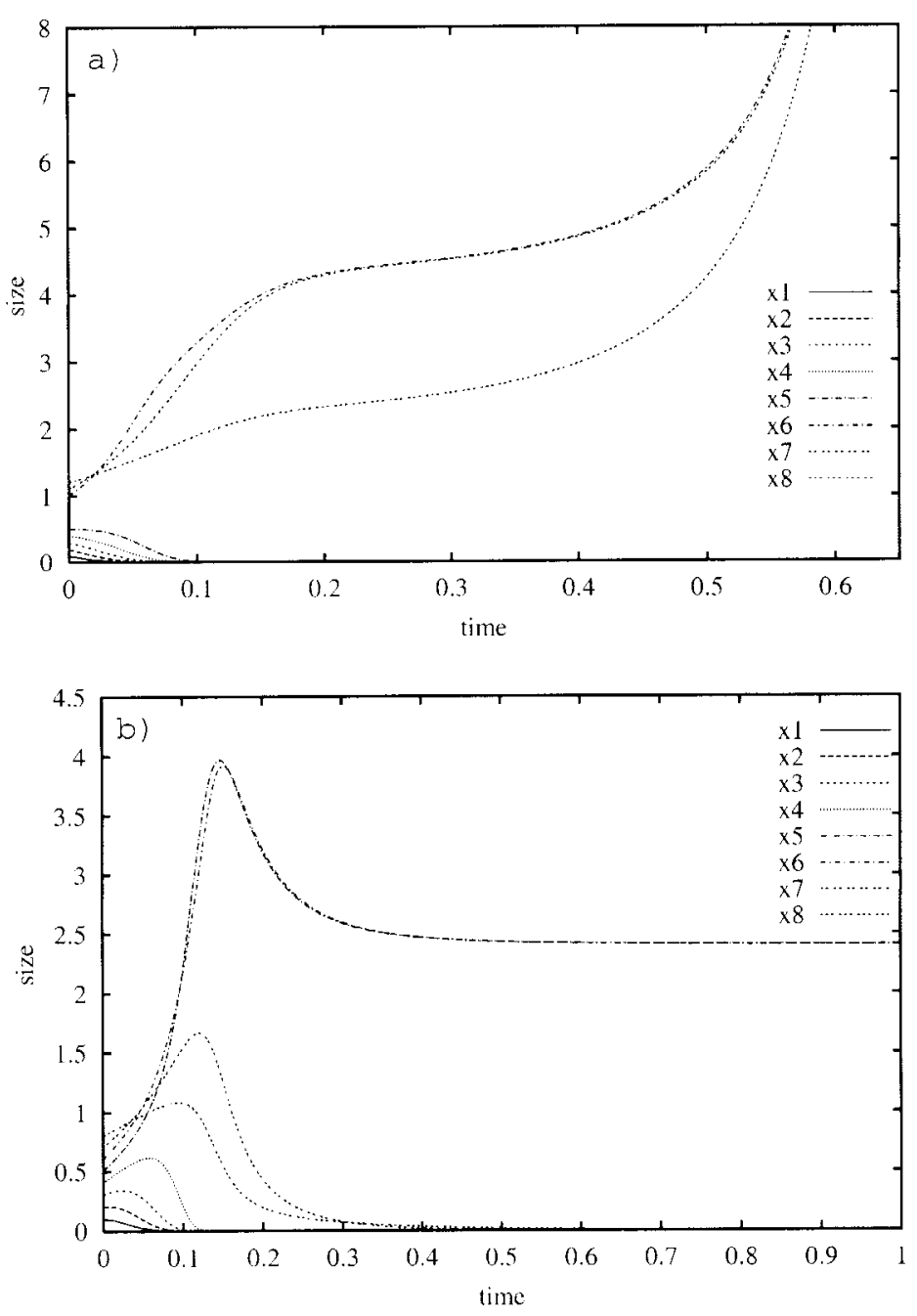

FIGURE 4 Simulation for the general situation where follicles interact with different initial sizes and ages for either three or two follicles selected. The parameters are $K=5.0, D=0.5, \mu=0.2, M_{1}=2.9, M_{2}=3.9$, and the initial age distribution is uniformly decreasing from $y_{10}=8.0$ to $y_{8_{1}}=1.0$. (a) The initial sizes are $x_{10}=0.1, \ldots, x_{50}=0.5, x_{6,9}=1.0, x_{7_{0}}=1.1, x_{8_{10}}=1.2$. Ovulating follicles $x_{6}$ and $x_{7}$ grow faster than the initial largest one $x_{8}$. (b) Initial sizes have the same value as in Fig. 1b. Follicles $x_{5}$ and $x_{6}$ grow till a pre-ovulatory size, whereas the two largest follicles, $x_{7}$ and $x_{8}$, atrophy and die.

have

$$
\frac{\mathrm{d} \tau^{\prime}}{\mathrm{d} X}<\frac{1}{(\lambda-D)} 1 / X
$$

Hence,

$\lim _{\tau^{\prime} \rightarrow \infty} t\left(\tau^{\prime}\right)=\int_{0}^{\infty} \frac{\mathrm{d} \tau^{\prime}}{y\left(\tau^{\prime}\right) \delta\left(X\left(\tau^{\prime}\right)\right)}<\frac{1}{y_{c}(\lambda-D)} \int_{X(0)}^{X(\infty)} \frac{\mathrm{d} X}{X \delta(X)}$

Thus, if $\delta(X)$ grows faster than $X^{\epsilon}$ for some $\epsilon>0$, the above integral is finite and $t\left(\tau^{\prime}\right)$ tends to a finite value $T$ as $\tau^{\prime} \rightarrow \infty$. Hence, $\tau^{\prime}$ goes to infinity in finite time, corresponding to ovulation in finite time.

When considering all follicles growing with the same initial age, but different size, the symmetry of Lacker's original model is still maintained. Thus, it is possible to reproduce similar numerical examples for this case as in Fig. 1. The only difference is that for the anovulatory situation, selected follicles do not reach a steady state but slowly deteriorate due to their ageing capacity. 

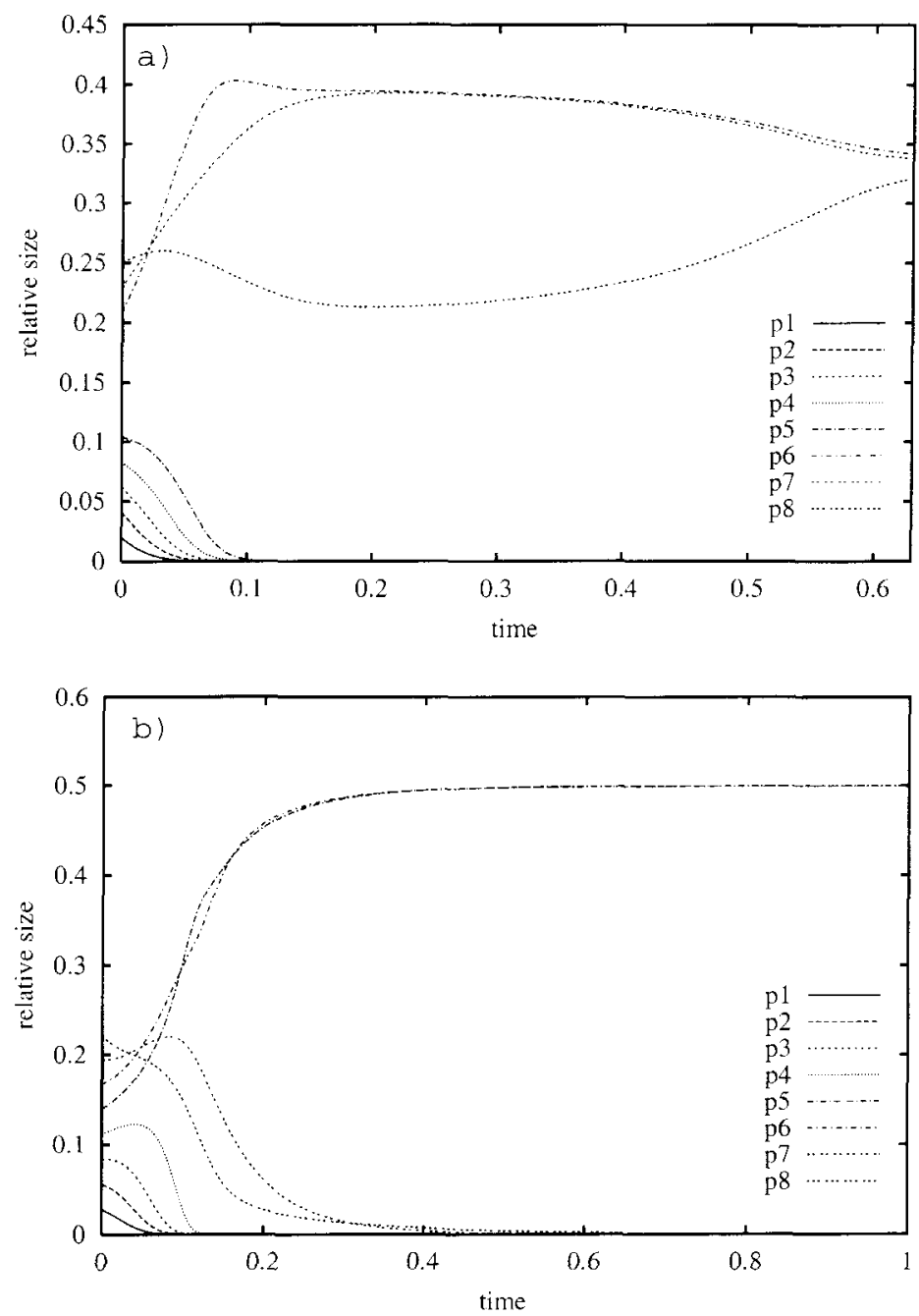

FIGURE 5 Simulation for follicles relative sizes interacting with different initial sizes and ages corresponding to Fig. 4. (a) The three largest follicles tend to the same equilibrium point $1 / 3$, and (b) the follicles relative maturities, $p_{5}$ and $p_{6}$, tend to a fixed value $1 / 2$.

When follicles interact with different size but same age, the model still regulates the number of pre-ovulatory follicles. Anovulation occurs in a more realistic way where follicles sizes do not remain constant indefinitely. Moreover, it is still possible to predict which follicles are selected from the initial size distribution. This predisposition is due to the strong follicle size hierarchy still present.

\section{DYNAMICS OF FOLLICLES WITH DIFFERENT SIZE AND AGE}

Let us now consider $N$ follicles interacting with different initial maturities (sizes) and ages. This means we are to analyze the original system (Eq. (4)). In this case, it is not possible to separate the dynamics in order to obtain a gradient system on the unit sphere. Hence, let us start by investigating some numerical 
examples for the parameter values used in previous simulations.

By an appropriate choice of initial ages and the same parameter values used in Fig. 1, it is possible to select follicles that are amongst the largest, yet they are not necessarily the largest (see Fig. 4). Although we can still predict the number of selected follicles, we are able to break the hierarchy for the selection mechanism.

Some evidence that system (Eq. (18)), for the space of follicles relative sizes, tend to the fixed point $\bar{p}_{M}$ is given in Fig. 5. Selected follicles approach to the same fixed equilibrium value in each case. Although it is not possible to determine which are the ones reaching preovulatory maturity, the system appears to be stable in terms of the pre-ovulation rate.

It is not possible to transform the interaction dynamics of system (Eq. (18)) into a gradient system in order to investigate the stability around $\bar{p}_{M}$. We then develop a linear stability analysis around a specific orbit. This kind of analysis is usually developed around a fixed equilibrium point. However, the principal goal of this section is to apply these ideas for the case of a non-autonomous system. Where, instead of a fixed equilibrium point, there is a particular orbit of interest.

The corresponding interaction dynamics and intensity dynamics for system (Eq. (4)) is

$$
\frac{\mathrm{d} p_{i}}{\mathrm{~d} \tau}=p_{i}\left[y_{i} \rho(X)+y_{i} \xi\left(p_{i}\right)-\rho(X) \sum_{j=1}^{N} y_{j} p_{j}-\sum_{j=1}^{N} y_{j} p_{j} \xi\left(p_{j}\right)\right]
$$

$$
\begin{aligned}
& \frac{\mathrm{d} y_{i}}{\mathrm{~d} \tau}=-\frac{\mu}{\delta(X)} y_{i} \\
& \frac{\mathrm{d} X}{\mathrm{~d} \tau}=X\left[\rho(X) \sum_{j=1}^{N} y_{j} p_{j}-\sum_{j=1}^{N} y_{j} p_{j} \xi\left(p_{j}\right)-\frac{\mu}{\delta(X)}\right] .
\end{aligned}
$$

Notice that Eq. (18a) depends explicitly on $X$, which does not occur in neither the interaction equation obtained from Lacker's model nor in Eq. (15a). For system (Eq. (15)), $X$ does not appear explicitly in Eq. (15a), but rather indirectly through $y$. However, by rescaling such equation, we have eliminated $y$, and analyzed the interaction equation independently of $X$.

By simplifying system (Eq. (18)) when $y_{i}=y_{j}=y$, we obtain the same interaction dynamics as in system (Eq. (15)). Hence, Eq. (18a) is a direct generalisation of Eq. (15a). For the interaction dynamics of system (Eq. (15)), the equilibrium point $\bar{p}_{M}$ given in Eq. (17) is stable. Solutions of system (Eq. (15)) lie along the lines of symmetry of the $M$-dimensional coordinate hyperplane in the $N$-dimensional space of follicles ages. Therefore, we consider $\bar{y} s=\underbrace{(y, \ldots, y)}_{N}$ for any $y>$ 0 , such that $\left(\bar{p}_{M}, \bar{y}_{s}\right)$ is the orbit around which the stability analysis of system (Eq. (18)) is developed. Since Eq. (18a) depends explicitly on $X$, it is harder to develop a linear analysis for this system than for system (Eq. (4)). Hence, we locally analyze the stability of system (Eq. (4)) around the orbit corresponding to $\left(\bar{p}_{M}, \bar{y}_{S}\right)$.

Note that for $\bar{p}_{M}$ given in Eq. (17), $p_{i}=1 / M$ implies that $x_{i} / X=1 / M$. Therefore, $x_{i}=X / M$ for all $i=$ $1, \ldots, M$ and $x_{i}=0$ for all $i=M+1, \ldots, N$ leads to

$p_{e}=\left(x_{i}, X, y\right)=\left\{\begin{array}{cllc}(X / M, X, y) & \forall i & = & 1, \ldots, M \\ (0, X, y) & \forall i & = & M+1, \ldots, N\end{array}\right.$

for any $X>0$ and any $y>0$. This is the orbit around which a linear stability analysis is developed for system (Eq. (4)).

\section{Linear Stability Analysis of the System of Follicles Different in Size and Age}

By substituting the explicit formula of $g\left(x_{i}, X\right)$ given in (Eq. (3)) into system (Eq. (4)), we obtain

$\frac{\mathrm{d} x_{i}}{\mathrm{~d} t}=y_{i} x_{i}\left[K-D\left(X-M_{1} x_{i}\right)\left(X-M_{2} x_{i}\right)\right]-\mu x_{i}=G_{1}$,

$\frac{\mathrm{d} y_{i}}{\mathrm{~d} t}=-\mu y_{i}=G_{2}$,

where $G_{k}=G_{k}\left(x_{i}, X, y_{i}\right)$ for all $k=1,2$. 
Let $\tilde{x}=\left(\tilde{x}_{1}, \ldots, \tilde{x}_{N}\right)$ and $\tilde{y}=\left(\tilde{y}_{1}, \ldots, \tilde{y}_{N}\right)$ so that $\varepsilon=$ $(\tilde{x}, \tilde{y})$ is a small perturbation around $p_{e}$ given in Eq. (19). The first order Taylor expansion then is

$$
F(\bar{x}+\tilde{x}, \bar{y}+\tilde{y})=F(\bar{x}, \bar{y})+D_{(x, y)} F \varepsilon+o\left(\|\varepsilon\|^{2}\right)
$$

where, $F=\overbrace{(G, \ldots, G)}^{N}$ is the symmetric vector field of the system and $G=\left(G_{1}, G_{2}\right)$. For the corresponding Jacobian, $J=D_{(\bar{x} \bar{y})} F$, we need

$$
\begin{aligned}
\frac{\mathrm{d} G_{k}}{\mathrm{~d} x_{i}} & =\frac{\partial G_{k}}{\partial x_{i}}+\frac{\partial G_{k}}{\partial X} \cdot \frac{\partial X}{\partial x_{i}} \\
\frac{\mathrm{d} G_{k}}{\mathrm{~d} y_{i}} & =\frac{\partial G_{k}}{\partial y_{i}}
\end{aligned}
$$

for $k=1,2$ and $i=1, \ldots, N$. Therefore, $J$ is a $2 N \times$ $2 N$ matrix such that

$$
J=\left[\begin{array}{ll}
\frac{\partial G_{1}}{\partial x}+\frac{\partial G_{1}}{\partial X} & \frac{\partial G_{1}}{\partial y} \\
\frac{\partial G_{2}}{\partial x}+\frac{\partial G_{2}}{\partial X} & \frac{\partial G_{2}}{\partial y}
\end{array}\right]
$$

When computing the various terms and evaluating them on $p_{e}$, the non-zero ones for $i=j$ are

(a) $i=1, \ldots, M$

$$
\begin{aligned}
& \left.\frac{\partial G_{1}}{\partial x_{i}}\right|_{p e}=y\left[K-D X^{2}+2 D\left(M_{1}+M_{2}\right) \frac{X^{2}}{M}\right. \\
& \left.-3 D M_{1} M_{2} \frac{X^{2}}{M^{2}}\right]-\mu=a_{1}, \\
& \left.\frac{\partial G_{1}}{\partial X}\right|_{p_{e}}=\frac{y D X^{2}}{M^{2}}\left[M_{1}+M_{2}-2 M\right]=b_{1}, \\
& \left.\frac{\partial G_{1}}{\partial y_{i}}\right|_{p e}=\frac{X}{M}\left[K-D X^{2}+D\left(M_{1}\right.\right. \\
& \left.\left.\quad+M_{2}\right) \frac{X^{2}}{M}-D M_{1} M_{2} \frac{X^{2}}{M^{2}}\right]=c, \\
& \left.\frac{\partial G_{2}}{\partial y_{i}}\right|_{p e}=-\mu
\end{aligned}
$$

(b) $i=M+1, \ldots, N$

$$
\begin{aligned}
& \left.\frac{\partial G_{1}}{\partial x_{i}}\right|_{p e}=y\left[K-D X^{2}\right]-\mu=a_{2}, \\
& \left.\frac{\partial G_{2}}{\partial y_{i}}\right|_{p e}=-\mu .
\end{aligned}
$$

For $i \neq j$, the non-zero terms are

(a) $i, j=1, \ldots, M$

$$
\left.\frac{\partial G_{1}}{\partial X}\right|_{p e}=b_{1}
$$

(b) $i=1, \ldots, M$ and $j=M+1, \ldots, N$

$$
\left.\frac{\partial G_{1}}{\partial X}\right|_{p e}=b_{1} .
$$

The resulting Jacobian evaluated on $p_{e}$ is the block matrix

$$
\begin{array}{c|c|c|c}
C & b_{1} U & c I_{M, M} & 0 \\
\hline 0 & a_{2} I_{N-M, N-M} & 0 & 0 \\
\hline 0 & -\mu I_{N, N}
\end{array}
$$

where, $C$ is the $(M \times M)$ circulant matrix

$$
C=\operatorname{circ} \underbrace{\left(a_{1}+b_{1}, b_{1}, \ldots, b_{1}\right)}_{M}
$$

This means that for all $i=1, \ldots, M$ of $C$, the $i$ th row, for $i>1$, is obtained by shifting the $(i-1)$ th row one entry to the right. The first row is given by the $1 \times M$ vector, $\left(a_{1}+b_{1}, b_{1}, \ldots b_{1}\right)$. Matrix $U$ is an $M \times$ $(N-M)$ matrix with all its entries equal to one, and $I_{n, m}$ is the identity matrix of size $n \times m$. Since $\left.J\right|_{p e}$ is an upper triangular block matrix, it is possible to analytically compute its eigenvalues. The set of such eigenvalues is referred to as the spectrum of $\left.J\right|_{p e}$ and is given by

$\operatorname{Spec}\left(\left.J\right|_{p e}\right)=$

$\left[\operatorname{Spec}(C), \operatorname{Spec}\left(a_{2} I_{N-M, N-M}\right), \operatorname{Spec}\left(-\mu I_{N, N}\right)\right]$ 
In particular, $\operatorname{Spec}(C)$ is given by,

$$
\begin{aligned}
& \lambda_{1}=a_{1}+M b_{1} \quad \text { with multiplicity } 1 \\
& \lambda_{2}=a_{1} \quad \text { with multiplicity } M-1,
\end{aligned}
$$

while $\operatorname{Spec}\left(a_{2} I\right)$ and $\operatorname{Spec}(-\mu I)$ give

$$
\begin{aligned}
& \lambda_{3}=a_{2} \quad \text { with multiplicity } N-M \text { and } \\
& \lambda_{4}=-\mu \quad \text { with multiplicity } N .
\end{aligned}
$$

When substituting the values of $a_{1}, b_{1}$ and $a_{2}$ from Eqs. (27)) and (Eq. (23)), and using the value of $\gamma$ given in (Eq. (6)), we get

$$
\begin{aligned}
\lambda_{1}= & y\left[K+3 D \gamma X^{2}\right]-\mu \\
\lambda_{2}= & y\left[K+D\left(\gamma+\frac{\left(M_{1}+M_{2}\right)}{M}-\frac{2 M_{1} M_{2}}{M^{2}}\right) X^{2}\right] \\
& -\mu
\end{aligned}
$$

$$
\begin{aligned}
& \lambda_{3}=y\left[K-D X^{2}\right]-\mu=a_{2} \\
& \lambda_{4}=-\mu .
\end{aligned}
$$

Note that $\lambda_{1}, \lambda_{2}$ and $\lambda_{3}$ are time dependent. Considering $y(t)=y_{0} \mathrm{e}^{-\mu t}$ and $X(t) \approx 1 / \sqrt{T-t}$, where $T$ is the fixed ovulation time, we have

$$
\begin{aligned}
& \lambda_{1} \approx K y_{0} \mathrm{e}^{-\mu t}+\frac{3 D \gamma y_{0}}{\mathrm{e}^{\mu t}(T-t)}-\mu \\
& \lambda_{2} \approx K y_{0} \mathrm{e}^{-\mu t}+\frac{D b y_{0}}{e^{\mu t}(T-t)}-\mu \\
& \lambda_{3} \approx K y_{0} \mathrm{e}^{-\mu t}-\frac{D y_{0}}{\mathrm{e}^{\mu t}(T-t)}-\mu,
\end{aligned}
$$

where $b=\left(\gamma+\frac{\left(M_{1}+M_{2}\right)}{M}-\frac{2 M_{1} M_{2}}{M^{2}}\right)$. Hence, as $t \rightarrow T$

$$
\begin{aligned}
& \lambda_{1} \rightarrow \frac{3 D \gamma y_{0}}{(T-t)} \rightarrow+\infty \\
& \lambda_{2} \rightarrow \frac{D b y_{0}}{(T-t)} \rightarrow-\infty \\
& \lambda_{3} \rightarrow \frac{-D y_{0}}{(T-t)} \rightarrow-\infty
\end{aligned}
$$

as long as $\gamma>0, b<0$ (see Fig. 6a).

When $\gamma<0, \lambda_{k}(t) \rightarrow-\mu$ for all $k=1,2,3,4$ since $X(t) \rightarrow 0$ and $y(t) \rightarrow 0$, see Fig. 6 b.

Notice that in Fig. 6b, the three eigenvalues are not all always negative. For this figure, the eigenvalues were evaluated for the anovulatory situation where the parameter values are the same as in Fig. 1. However, by changing the initial size distribution so that one follicle is relatively larger than the rest, there are also two anovulatory follicles as we see in Fig. 7.

By the time the two pre-ovulatory follicles have been already selected, $\lambda_{3}(t)>0$ (see Figs. $6 \mathrm{~b}$ and 7 ). To have a positive eigenvalue when solutions have already converged may be intuitively contradictory. Thus, let us compute the corresponding eigenvector for each eigenvalue to determine expanding or contracting directions.

To find such eigenvectors along the orbit $X(t)$, let us solve the system

$$
\begin{gathered}
{[C-\lambda I] \bar{x}_{M}+b_{1} U \bar{x}_{N-M}+c I \bar{y}_{M}=0} \\
\left(a_{2}-\lambda\right) I \bar{x}_{N-M}=0 \\
(-\mu-\lambda) I \bar{y}=0
\end{gathered}
$$

where, $\bar{x}_{M}$ and $\bar{x}_{N-M}$ are vectors obtained, respectively, from the first $M$ and the remaining $N-M$ coordinates of $\bar{x}$. The vector $\bar{y}_{M}$ is obtained from the first $M$ entries of $\bar{y}$.

For $\lambda_{1}$, we get $\bar{x}_{N-M}=0$ and $\bar{y}=0$ implying that $\bar{y}_{M}=0$. The corresponding eigenvector for $\lambda_{1}$ of matrix $C$ is such that, $\bar{x}_{M}=\bar{u}_{M}$ where $\bar{u}_{M}$ has all of its $M$ coordinates equal to one (Bellman, 1960). This 

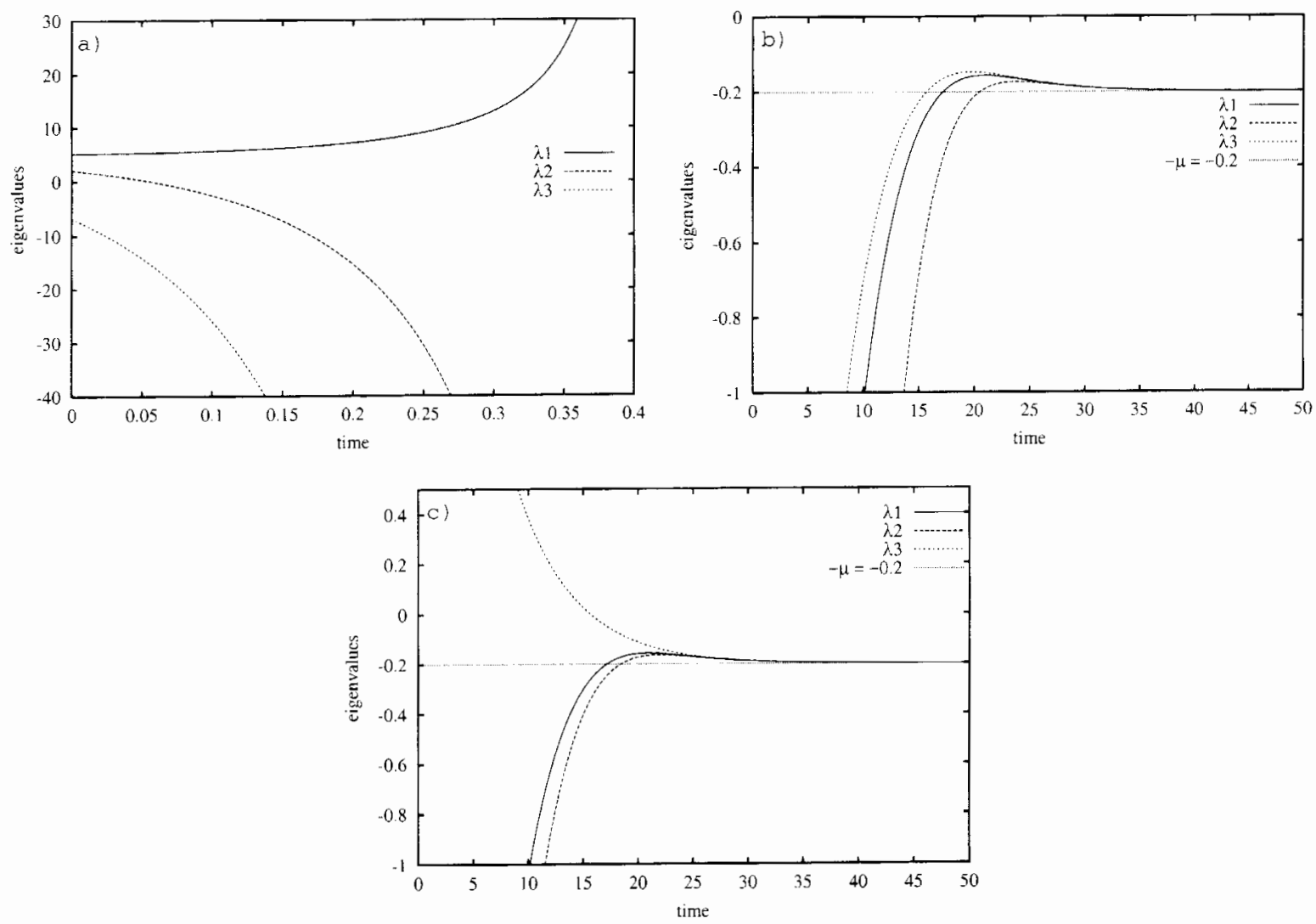

FIGURE 6 (a) Eigenvalues evaluated for function $X(t)$ given in (Eq. (9)). Where, $y_{0}=1.0$ and parameter values $K, D, \mu, M_{1}$ and $M_{2}$ are the same as for the ovulating case of Fig. 4. The initial value $X_{0}=4.8$ is the sum of the initial follicles sizes given in Fig. 4 . (a) $M=3$ so that $\gamma>0$, which implies $\lambda_{1}(t) \rightarrow \infty$, and $\lambda_{k}(t) \rightarrow-\infty$ for $k=2,3$ as $t \rightarrow T$. In Figs. b) and c) $\gamma<0$, so that $\lambda_{k} \rightarrow-\mu=-0.2$ as $t \rightarrow \infty$ for all $k=1,2,3$. (b) Initial size distribution is such that $M=2$, and all of the three eigenvalues are always negative. (c) Initial size distribution is such that $M=1$, and $\lambda_{3}>0$ at the beginning of the cycle.

means that such a vector gives the direction of the $M$ identical non-zero follicles sizes. Let us define $V_{1}=$ $\left\{\beta \bar{u}_{M}: \beta \in \mathbb{R}\right\} \subseteq \mathbb{R}^{N}$. For $\lambda_{2}$, we get $N-M$ eigenvectors $\bar{v}=\bar{x}_{M}$ such that,

$$
\sum_{k=1}^{M} v_{k}=0
$$

(Bellman, 1960). Observe that $\bar{v} \perp \bar{u}_{M}$ for all $\bar{v}$, so we define

$$
V_{1}^{\perp}=\left\{\bar{v}: \sum_{k=1}^{M} v_{k}=0\right\} .
$$

For $\lambda_{3}$, we see that from (Eq. (26c)) $\bar{y}=0$, and from (Eq. (26b)), $\bar{x}_{N-M} \neq 0$. Suppose $\bar{x}_{N-M}=\bar{u}_{N-M}$, where $\bar{u}_{N-M}$ is the vector for which all of its $N-M$ entries are equal to one. Then, $\bar{x}_{M} \in V_{1}$, i.e. $\bar{x}_{M}=$ $\beta \bar{u}_{M}$, where

$$
\beta=-\frac{(N-M) b_{1}}{\left(\lambda_{1}-\lambda_{3}\right)} .
$$

Substituting the values of $\lambda_{1}$ and $\lambda_{3}$ from (Eq. (25)), where $\lambda_{1} \neq \lambda_{3}$ for all $X$ and $y$, and substituting the value of $b_{1}$ from (Eq. (22)), we get

$$
\beta=-\frac{(N-M)\left(M_{1}+M_{2}-2 M\right)}{M(3 \gamma+1)} .
$$

We thus see that $\beta$ does not depend on $t$. Let us suppose $\bar{x}_{N-M}=\bar{v}$, such that $\bar{v} \in V_{1^{\prime}}^{\perp}$, where $V_{1^{\prime}}^{\perp}$, is of 


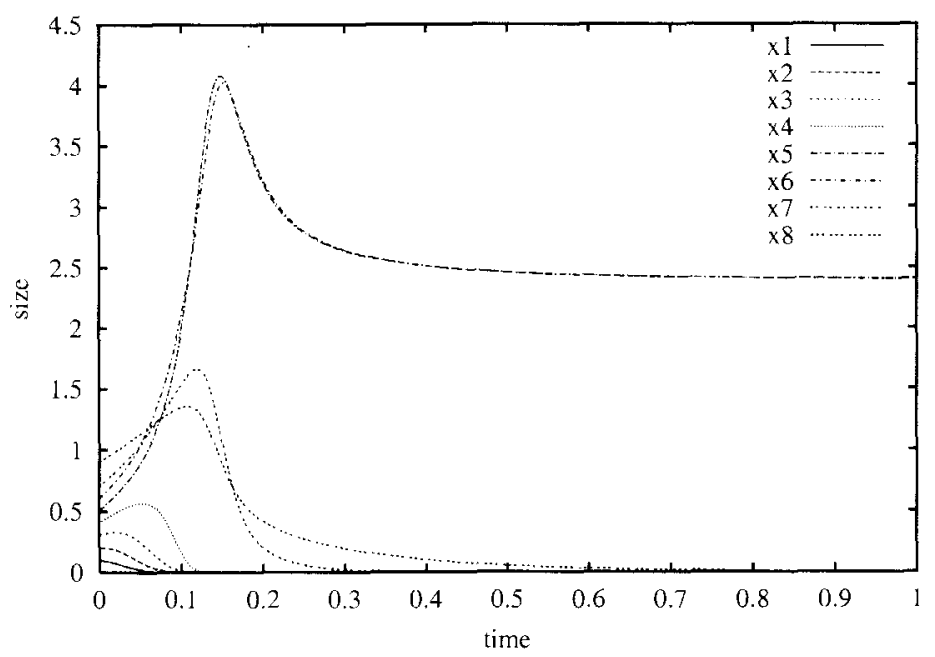

FIGURE 7 Numerical simulation of eight interacting follicles for parameters, $K=5.0, D=0.5, \mu=0.2, M_{1}=2.9, M_{2}=3.9$, and initial conditions $y_{i 0} \forall i=1, \ldots, 8$ as in Fig. $4 \mathrm{~b}$. The initial maturity distribution is such that follicle $x_{8}$ initial size is relatively larger than the rest seven follicles. Follicles $x_{5}$ and $x_{6}$ are the ones reaching the same pre-ovulatory maturity, while the reminding five follicles, $x_{1}, \cdots, x_{4}, x_{7}$ and $x_{8}$, atrophy and die.

dimension $N-M$, i.e. $\sum_{k=1}^{N-M} v_{k}=0$, so that $\bar{u}_{N-M} \perp$ $\bar{v}$. Then, from system (Eq. (26)), we get $[C-$ $\left.\lambda_{3} I\right] \bar{x}_{M}=0$, implying that $\bar{x}_{M}=0$.

Since for the eigenvectors corresponding to $\lambda_{1}, \lambda_{2}$ and $\lambda_{3}$, we have $\bar{y}=0$, we then analyze their behavior only in the space of sizes $\bar{x} \in \mathbb{R}^{N}$. Thus, for matrix

$$
A=\left[\begin{array}{l|l}
C & b_{1} U \\
\hline 0 & a_{2} I
\end{array}\right]
$$

the eigenvectors are,

$$
\begin{aligned}
& \bar{v}_{1}^{T}=\left(\bar{u}_{M}^{T}, \overline{0}_{N-M}^{T}\right), \\
& \bar{v}_{2}^{T}=\left(v^{T}, \overline{0}_{N-M}^{T}\right), \text { such that } v \in V_{1}^{\perp}, \\
& \bar{v}_{3}^{T}=\left(\beta \bar{u}_{M}^{T}, \bar{u}_{N-M}^{T}\right), \\
& \bar{v}_{3^{\prime}}^{T}=\left(\overline{0}_{M}^{T}, \bar{v}^{T}\right), \text { such that } \bar{v} \in V_{1^{\prime}}^{\perp}
\end{aligned}
$$

Note that none of them depend on time. These eigenvectors give a basis for the whole space of follicles sizes. In particular, $\bar{v}_{1}$ gives the direction of the $M$ identical non-zero follicles. Changes in the direction of $\bar{v}_{1}$ correspond to changes in the follicles total size or oestradiol concentration $X$. On the other hand, $\bar{v}_{2}$ corresponds to the direction of the $M-1$. vectors perpendicular to $\bar{v}_{1}$, and any change in $\bar{v}_{2}$ does not affect the dynamics of $X$. Finally, $\bar{v}_{3}$ and $\bar{v}_{3}$ give two different directions for the remaining $N-M$ follicles. Changes in $\bar{v}_{3}$ give the dynamics of the total size of those follicles, and any change in $\bar{v}_{3^{\prime}}$ does not change $X$.

The sign of $\lambda_{1}$ determines whether we are in an ovulatory $\left(\lambda_{1}>0\right)$ or anovulatory $\left(\lambda_{1}<0\right)$ situation since its corresponding eigenvector, $\tilde{v}_{1}$, points in the direction of the total oestradiol solution $X(t)$. This is equivalent to the dynamics of the $M$ follicles having the same size or oestradiol production $X / M$. On the other hand, the sign of $\lambda_{2}$ determines whether orbits approach to or move away from the line along the direction of $\bar{v}_{1}$. And finally, the sign of $\lambda_{3}$ indicates the behavior in the remaining $N-M$ subspace of follicles with small initial size. Dynamics on the direction of $\bar{v}_{3}$ gives the behavior when all of the $N-M$ smallest follicles have the same initial size, whereas $\bar{v}_{3^{\prime}}$ describes the dynamics in the direction perpendicular to $\bar{v}_{3}$.

To study the local stability of the perturbation, let us apply the original linearised system to each of the eigenvectors, i.e. let us compute $\mathrm{d} \bar{v}_{k} / \mathrm{d} t=A \bar{v}_{k}$ for all $k=1,2,3,3^{\prime}$, and see how each $\bar{v}_{k}$ varies along $X(t)$. 
From the eigenvectors given in Eq. (28), we obtain the following system,

$$
\begin{aligned}
& \frac{\mathrm{d} \bar{v}_{1}}{\mathrm{~d} t}=A \bar{v}_{1}=\lambda_{1} \bar{v}_{1} \\
& \frac{\mathrm{d} \bar{v}_{2}}{\mathrm{~d} t}=A \bar{v}_{2}=\lambda_{2} \bar{v}_{2}
\end{aligned}
$$

$$
\begin{gathered}
\frac{\mathrm{d} \bar{v}_{3}}{\mathrm{~d} t}=A \bar{v}_{3} \\
=\left(\begin{array}{c}
\lambda_{1} \beta \bar{u}_{M} \\
\lambda_{3} \bar{u}_{N-M}
\end{array}\right)+(N-M) b_{1}(t)\left(\begin{array}{c}
\bar{u}_{M} \\
0
\end{array}\right) \\
\frac{\mathrm{d} \bar{v}_{3^{\prime}}}{\mathrm{d} t}=A \bar{v}_{3^{\prime}}=\lambda_{3} \bar{v}_{3^{\prime}} .
\end{gathered}
$$

For the non-zero coordinates of $\bar{v}_{1}, \bar{v}_{2}, \bar{v}_{3}$ and $\bar{v}_{3^{\prime}}$, we obtain

$$
\begin{array}{r}
{\left[\dot{v}_{1}\right]_{j}=\lambda_{1}\left[v_{1}\right]_{j} \quad \forall j=1, \ldots, M} \\
{\left[\dot{v}_{2}\right]_{j}=\lambda_{2}\left[v_{2}\right]_{j} \quad \forall j=1, \ldots, M} \\
{\left[\dot{v}_{3^{\prime}}\right]_{j}=\lambda_{3}\left[v_{3^{\prime}}\right]_{j} \quad \forall j=M+1, \ldots, N}
\end{array}
$$

and,

$\left[\dot{v}_{3}\right]_{j}=\left\{\begin{array}{cc}\lambda_{1}\left[v_{3}\right]_{j}+(N-M) b_{1}(t)\left[v_{1}\right]_{j} & \forall j=1, \ldots, M \\ \lambda_{3}\left[v_{3}\right]_{j} & \forall j=M+1, \ldots, N .\end{array}\right.$

The solution for eigenvectors $\bar{v}_{1}$ and $\bar{v}_{2}$ is given by,

$$
\bar{v}_{k}(t)=\bar{v}_{k}(0) \exp \left(\int_{0}^{t} \lambda_{k}(s) \mathrm{d} s\right)
$$

for $k=1,2$. Similarly, for $\bar{v}_{3^{\prime}}$

$$
\bar{v}_{3^{\prime}}(t)=\bar{v}_{3^{\prime}}(0) \exp \left(\int_{0}^{t} \lambda_{3}(s) \mathrm{d} s\right)
$$

and for the $j=M+1, \ldots, N$ entries of $\bar{v}_{3}$, we also have

$$
\left[v_{3}\right]_{j}(t)=\left[v_{3}\right]_{j}(0) \exp \left(\int_{0}^{t} \lambda_{3}(s) \mathrm{d} s\right)
$$

From Eqs. (30a) and (31a), if $\lambda>0, \lambda_{1}(t)>0$ implying that the first $M$ coordinates of $\bar{v}_{1}$ and $\bar{v}_{3}$ grow to infinity. In contrast, the first $M$ coordinates of $\bar{v}_{2}$ converge to zero due to $\lambda_{2}(t) \rightarrow-\infty$. From Eqs. (30c) and (31b), the $N-M$ coordinates of $\bar{v}_{3^{\prime}}$ and $\bar{v}_{3}$, respectively, tend to zero as $\lambda_{3} \rightarrow-\infty$ (see Fig. 8a).

This means, on one hand, that the corresponding vectors in $V_{1}$ formed by the $M$ identical non-zero components of vectors $\bar{v}_{1}$ and $\bar{v}_{3}$, point towards the same direction in which the orbit grows to infinity on finite time, i.e. towards the direction given by $\bar{u}_{M}$. On the other hand, for the first $M$ components of vector $\bar{v}_{2}$, the corresponding vector which points in a direction perpendicular to that given by vectors in $V_{I}$, contracts with time. In other words, when $M$ different, but similar large follicles start the cycle, they will tend to the line along the direction given by $\bar{u}_{M}$ and then grow to infinity in finite time.

The $N-M$ non-zero coordinates of $\bar{v}_{3}$ and $\bar{v}_{3^{\prime}}$ describe the dynamics of initial small follicles. Thus, when small follicles start the cycle, they will tend towards the line in the direction of $\bar{v}_{3}$ and then tend to zero maturity.

When $\lambda<0$, the $M$ identical coordinates of the three different eigenvectors converge to zero since $\lambda_{\mathrm{k}} \rightarrow-\mu$ for all $k=1,2,3$ (see in Fig. 8b). For the particular example given in Fig. $6 \mathrm{c}$, where $\lambda_{3}(t)>0$ during the cycle, Fig. $8 \mathrm{c}$ shows that also the first $M$ coordinates of $\bar{v}_{1}$ and $\bar{v}_{3}$ tend to zero. This means that the dynamics along the line generated by $\bar{u}_{M}$ tends to zero. Furthermore, the $M$ non-zero coordinates of $\bar{v}_{2}$ which generate a vector perpendicular to $\bar{u}_{M}$ also tend to zero. Thus, whenever the cycle starts with $M$ different, but similar large follicles, they will tend to the same size and then decrease and die. For the dynamics of the $N-M$ coordinates of $\bar{v}_{3}$ since $\lambda_{3}(t)>0$ during the cycle, as it is seen in Fig. $6 \mathrm{c}$, the $N-M$ initially identical small follicles would grow to a large size. Nevertheless, although such coordinates grow to a very large value, they eventually tend 
to zero as expected since $\lambda_{3}(t) \rightarrow-\mu$ as $t \rightarrow \infty$ (see Fig. 8d). At the same time, the $N-M$ follicles that initiate the cycle with different small sizes will tend to the line in the direction given by $\overline{v_{3}}$ and then eventually tend to zero.

It remains to compute the eigenvectors for $\lambda_{4}=$ $-\mu$ corresponding to perturbations in $\bar{y}$. We then consider the whole $2 N$ dimensional space and solve system (Eq. (26)) for $\lambda_{4}$. From Eq. (26c), we get $\bar{y} \neq 0$, and from (Eq. (26b)), we get $\bar{x}_{N-M}=0$. To find the corresponding $\bar{x}_{M}$, we consider two cases:

(a) If $\bar{y}=\bar{u}_{N}$ (the vector for which its $N$ coordinates are equal to one) corresponding to the same perturbation to all ages, then $\bar{x}_{M} \in V_{1}$. Let $\bar{x}_{M}=$ $\alpha \bar{u}_{M}$ where,

$$
\alpha=\frac{c}{\lambda_{4}-\lambda_{1}}
$$

such that, $\lambda_{4} \neq \lambda_{1}$ for all $X>0$ and $y>0$. From the values of $c$ and $\gamma$ given in Eqs. (22) and (6), respectively, we get

$$
c=\frac{X}{M}\left[K+\gamma D X^{2}\right]
$$

From the eigenvalues given in Eq. (25), we obtain

$$
\alpha=-\frac{X(t)\left[K+\gamma D X(t)^{2}\right]}{y(t) M\left[K+3 \gamma D X(t)^{2}\right]}
$$

thus, $\alpha=\alpha(t)$. Therefore,

$$
\bar{v}_{4}^{T}=\left(\alpha(t) \bar{u}_{M}^{T}, \overline{\mathrm{O}}_{N-M}^{T} ; \bar{u}_{N}^{T}\right) \in \mathbb{R}^{2 N},
$$

which varies with time.

(b) If $\bar{y}=\bar{v} \in V_{1^{\prime \prime}}$, where $V_{\mathrm{I}^{\prime \prime}}^{\perp}=\left\{\bar{v}: \sum_{k=1}^{N} v_{k}=0\right\}$ such that $\bar{u}_{N} \perp \bar{v}$ for all $\bar{v} \in V_{l^{\prime \prime}}^{\perp}$. Let $\bar{v}^{T}=$ $\left(\bar{v}_{M}^{T}, \bar{v}_{N-M}^{T}\right)$, where $\bar{v}_{M}$ corresponds to the first $M$ entries of $\bar{v}$, and $\bar{v}_{N-M}$, the remaining $N-M$ coordinates. Then, from Eq. (26a) we get,

$$
\left[C-\lambda_{4} I\right] \bar{x}_{M}+c I \bar{v}_{M}=\bar{O}_{M},
$$

from which there are two sub-cases: (b.1) If $\bar{x}_{M} \in V_{1}$, then let $\bar{x}_{M}=\alpha^{\prime} \bar{u}_{M}$ for a given $\alpha^{\prime} \neq 0$. Thus, for $i=1, \ldots, M$ we get,

$$
\left(\lambda_{1}-\lambda_{4}\right) \alpha^{\prime}+c v_{i}=0 \text {. }
$$

From which,

$$
v_{i}=\frac{\left(\lambda_{4}-\lambda_{1}\right) \alpha^{\prime}}{c}=\beta^{\prime},
$$

meaning that $\bar{v}_{M}=\beta^{\prime} \bar{u}_{M}$, implying that $\bar{v}_{M} \in V_{1}$. The eigenvector then obtained is

$$
\bar{v}_{4^{\prime}}^{T}=\left(\alpha^{\prime} \bar{u}_{M}^{T}, \overline{0}_{M}^{T}, \beta^{\prime} \bar{u}_{M}^{T}, \bar{v}_{N-M}^{T}\right)
$$

(b.2) If $\bar{x}_{M} \in V_{1}^{\perp}$, the $i$ th equation in Eq. (26a) is

$$
\left(a_{1}-\lambda_{4}\right) x_{i}+c v_{i}=0
$$

Hence,

$$
v_{i}=\frac{\left(\lambda_{4}-\alpha_{1}\right) x_{i}}{c}
$$

implying that $\bar{v}_{M} \in V_{1}^{\perp}$, since $\sum_{i=1}^{M} x_{i}=0$, and

$$
\sum_{i=1}^{M} v_{i}=\frac{\lambda_{4}-a_{1}}{c} \sum_{i=1}^{M} x_{i}=0 .
$$

The eigenvector then is

$$
\bar{v}_{4^{\prime \prime}}^{T}=\left(\bar{x}_{M}^{T}, \bar{o}_{N-M}^{T}, \bar{v}_{M}^{T}, \bar{v}_{N-M}^{T}\right)
$$

where $\bar{x}_{M}, \bar{v}_{M} \in V_{1}^{\perp}$.

Let us discuss the behavior of $\bar{v}_{k}$ for the linearised dynamics $\dot{\vec{v}}_{k}=\left.J\right|_{p e} \vec{v}_{k:}$ for $k=4,4^{\prime}, 4^{\prime \prime}$ and the matrix $J \mid p_{e}$ given in Eq. (24). For the non-zero coordinates, we get the following system,

$$
\left[\dot{v}_{4}\right]_{j}=\left\{\begin{array}{cc}
\lambda_{1}\left[v_{4}\right]_{j}+c(t)\left[v_{1}\right]_{j} & \forall j=1, \ldots, M \\
\lambda_{4}\left[v_{4}\right]_{j} & \forall j=1, \ldots, N .
\end{array}\right.
$$



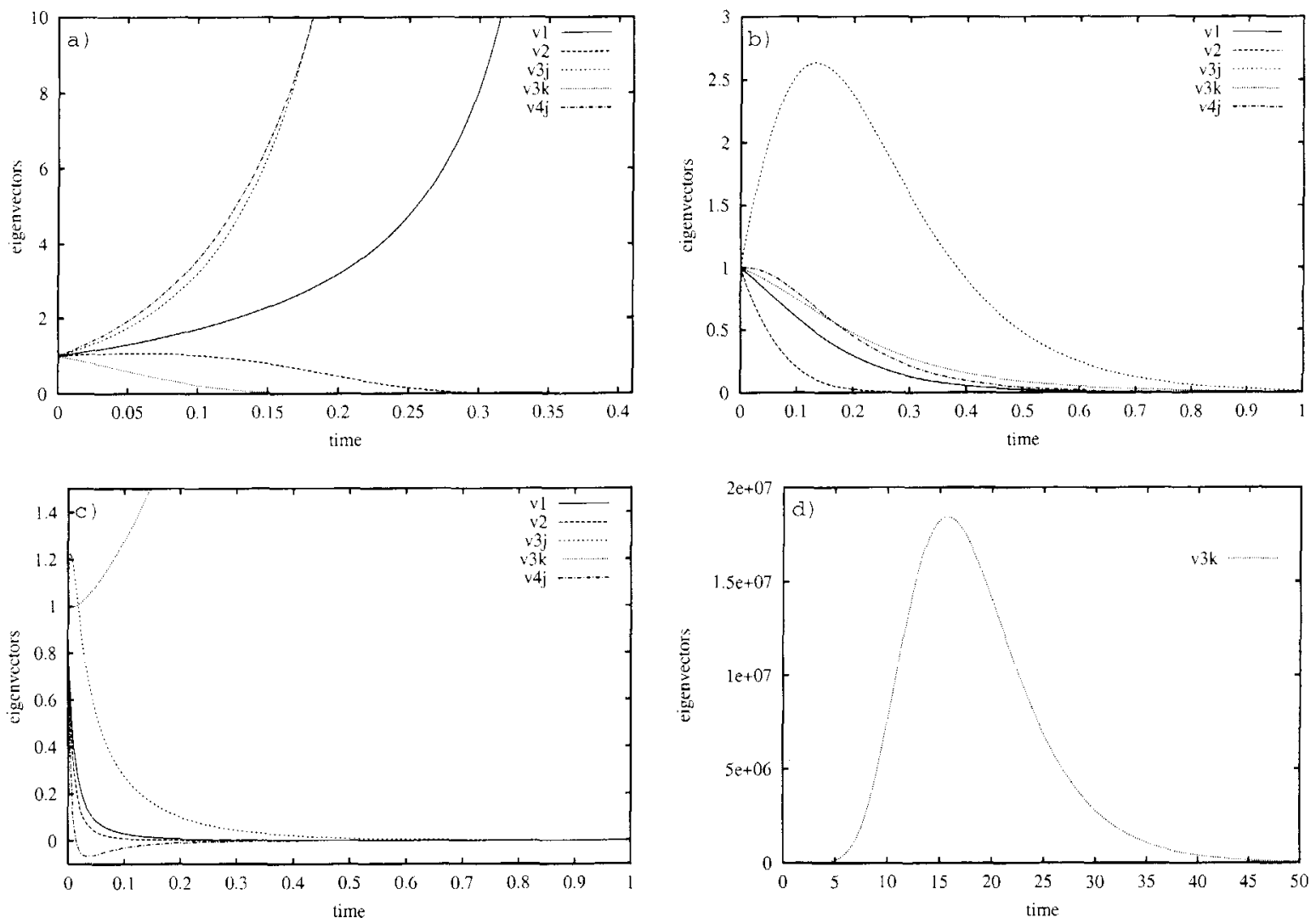

FIGURE 8 Eigenvectors dynamics. Graphs of $v_{1},\left[v_{3}\right]_{;}$and $\left[v_{4}\right]_{j}$, represent the behavior of $\bar{v}_{1}$, and the behavior of the first $M$ non-zero coordinates of $\bar{v}_{3}$ and $\bar{v}_{4}$, respectively. Furthermore, $v_{2}$ and $\left[v_{3}\right]_{k}$ represent the dynamics of $\bar{v}_{2}$ and the $N-M$ non-zero coordinates of $\bar{v}_{3}$. (a) When $\gamma>0$, eigenvectors correspond to eigenvalues shown in Fig. $6 \mathrm{a} . \bar{v}_{1},\left[v_{3}\right]_{j}$ and $\left[v_{4}\right]_{j}$ for $j=1, \ldots, M$ grow to infinity at a finite time, while $\bar{v}_{2}$ and $\left[v_{3}\right]_{k}$ for $k=M+1, \ldots, N$ tend to zero. (b) When $\gamma<0$, eigenvectors correspond to eigenvalues shown in Fig. 6b. (b) All of them tend to zero. (c) The $N-M$ coordinates of $\bar{v}_{3}$ grow. (d) Dynamics of the $N-M$ coordinates of $\bar{v}_{3}$ obtained from $\lambda_{3}$ computed for $\gamma<0$ shown in Fig. 6c. Although such coordinates increase to a large value, they eventually tend to zero since $\lambda_{3}(t) \rightarrow-\mu$ as $t \rightarrow \infty$.

For the non-zero coordinates of $\bar{v}_{k}$, where $k=$ $4^{\prime}, 4^{\prime \prime}$, we get

$$
\left[\dot{v}_{k}\right]_{j}= \begin{cases}\lambda_{4} v_{k_{j}} & \text { for all } j=1, \ldots, M \\ \lambda_{4} v_{k_{j}} & \text { for all } j=1, \ldots, N .\end{cases}
$$

Vectors $\overline{v_{4^{\prime}}}$ and $\bar{v}_{4^{\prime \prime}}$ are obtained by considering $\bar{y} \in V_{1^{\prime \prime}}^{\perp}$. We then have

$$
\bar{v}_{k}(t)=\bar{v}_{k}(0) \mathrm{e}^{-\mu t}
$$

for $k=4^{\prime}, 4^{\prime \prime}$. Meaning that within both subspaces, the one of follicular sizes and the one of follicular ages, the dynamics along those two directions is contractive.

Dynamics of coordinates $\left[v_{k}\right]_{j}$ for $j=1, \ldots, N$ and $k=4,4^{\prime}, 4^{\prime \prime}$, within the space of follicular ages is given by,

$$
\left[v_{k}\right]_{j}(t)=\left[v_{k}\right]_{j}(0) \mathrm{e}^{-\mu t}
$$

It is clear that for the subspace of follicles ages, the dynamics of all of the eigenvectors $\bar{v}_{k}$ tends exponentially to zero at rate $-\mu$. In particular, $\bar{y} \in$ $V_{1^{\prime \prime}}^{\perp}$ implies that when the cycle starts with follicles with different initial ages, their age values tend to the diagonal given by $\bar{y}=\bar{u}_{M}$. At the same time, all 
follicles ages tend to zero as the solution in the direction of $\bar{y}=\bar{u}_{M}$ also tends to zero.

As for the follicle sizes, this analysis tells us that when $\gamma>0$, the first $M$ coordinates of $\bar{v}_{4}$ tend to infinity in finite time (see Fig. 8a). For the first $M$ coordinates of vectors $\bar{v}_{4^{\prime}}$ and $\bar{v}_{4^{\prime \prime}}$, the dynamics tends to zero at the same rate $-\mu$. In contrast, when $\gamma<$ $0,\left[v_{4}\right]_{j}$ for $j=1, \ldots, M$ tend to zero as we see in Fig. $8 \mathrm{~b}$ and $\mathrm{c}$. Furthermore, for $\gamma<0$, the first $M$ entries of $\bar{v}_{4^{\prime}}$ and $\bar{v}_{4^{\prime \prime}}$ also tend to zero maturity. This means that the dynamics along the line generated by $\bar{u}_{M}$ is the same as the dynamics of the total amount of oestradiol $X$, and it is either ovulatory $(\gamma>0)$ or anovulatory $(\gamma<0)$.

So far, we have only developed a local linear stability analysis. Since our proof is about a trajectory and not about an equilibrium point, some special consideration should be taken for the second order term of the Taylor approximation (Eq. (21)). For the particular case of anovulation, convergence of such a term follows straight forwardly since $X(t) \rightarrow 0$ as $t \rightarrow \infty$. For the ovulatory case, this question is more delicate because of unboundedness of solutions. Proving nonlinear stability falls outside the scope of the present manuscript and will be left open for further study.

\section{Further Results}

New results from this model can be observed when initial conditions of system (Eq. (4)) are not similar. In the cases where there are either three follicles ovulating or two stuck follicles, drastic alterations in the selection process are obtained when significantly changing the initial conditions of the system. In this case, the largest follicles initial ages were reduced to a value much smaller than the ages of the remaining interacting follicles.

For the particular situation where previously, the largest three follicles would ovulate, Fig. 9a shows how such follicles become anovulatory, and eventually atrophy. Although these three follicles decrease in size, they are not atretic when we compare their decrease rate with smaller follicles $x_{1}-x_{5}$. From the separatrix initial value given in Eq. (10), observe that $X_{0}^{*}$ is inversely proportional to the initial age $y_{0}$. Thus, for the case of follicles starting the cycle with different ages, the "oldest", ones start growing with an initial size smaller than the minimum threshold size $X_{0}^{*}$ required to ovulate.

For this case, it is irrelevant that the largest follicles are able to ovulate in terms of their hormonal sensitivity. If two of them are old enough at the beginning of the cycle, they would not ovulate and would also obstruct other follicles from ovulating. Hence, the ageing model gives the possibility of getting the same number of follicles ovulating or arresting only by a significantly large change in the initial conditions of the system. This is another feature that Lacker's model is unable to exhibit since the number of ovulating follicles is always strictly larger than the number of stuck follicles. Incorporating the ageing factor into Lacker's original model suggests that such a decaying capacity may be the reason for PCO, when hormonal levels are adequate for a normal selection process.

We also observe that the relative maturity of the selected follicles $p_{7}$ and $p_{6}$ does not tend to the same fixed value as in Fig. 5. In contrast, those follicles tend to a different equilibrium point, whilst the largest follicle relative maturity, $p_{8}$, tends to zero (see Fig. $9 \mathrm{~b})$. This shows that we can only guarantee local stability for the model of follicles with different size and age.

For the anovulatory case, if the initial age of the two largest follicles is significantly reduced, it does not really affect the selection process. For this situation, Fig. 10a shows that follicles five and six are again selected and remain stuck, but the two largest follicles decrease much more slowly than in Fig. 5b. However, we would not consider follicles seven and eight as being anovulatory since they still decrease much faster than follicles five and six. Furthermore, Fig. 10b shows follicles five and six are still the selected ones. This agrees with the fact that for the anovulatory case, there is no minimum threshold required for follicles to be able to reach a preovulatory size. 

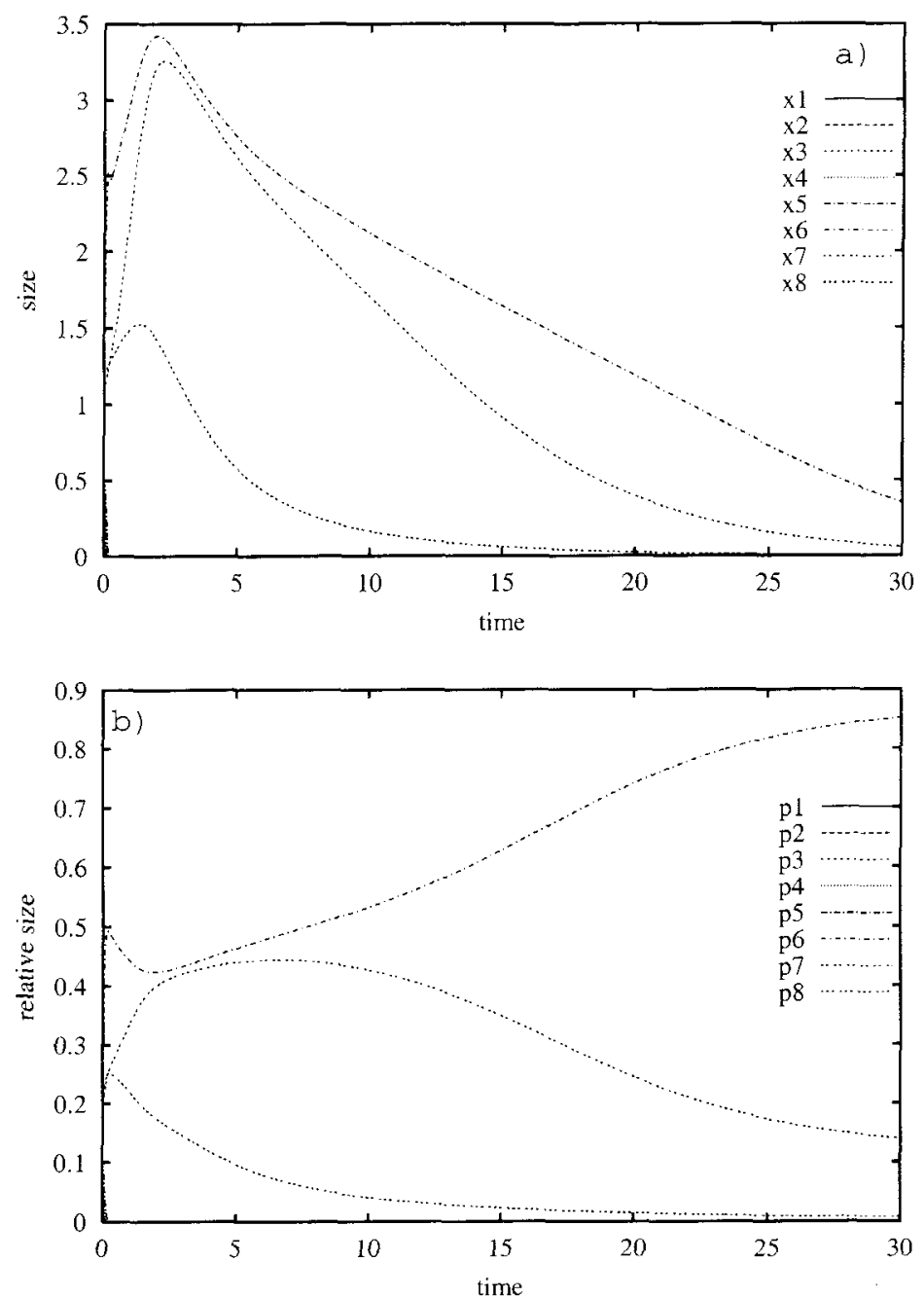

FIGURE 9 (a) Simulation of the ageing model where $K=5.0, D=0.5, \mu=0.2, M_{1}=2.9$, and $M_{2}=3.9$. Initial age values do not decrease uniformly as in previous examples, but $y_{1_{0}}=8.0, \ldots, y_{6}=3.0$ while, $y_{70}=0.2$ and $y_{8_{0}}=0.1$. The three largest follicles, $x_{6}, x_{7}$, and $x_{8}$ reach pre-ovulatory maturity, yet they start to atrophy so slowly that it appears they remain stuck in the ovary. Rather than ovulatory, these three follicles get stuck inside the ovary. (b) Numerical simulation of follicles relative oestradiol production corresponding to (a). Follicles with the largest relative maturity do not tend to the equilibrium value of $1 / 3$, instead follicle $p_{8}$ tends to zero and follicles $p_{6}$ and $p_{7}$ tend to different equilibrium points.

\section{DISCUSSION}

Implementation of the ageing variable complicates the mathematical expression of Lacker's system, but it is still possible to develop a relevant theoretical analysis, which is basically the new contribution of this paper. The theoretical and numerical analysis of Mariana et al.'s model developed here is able to give new tentative conclusions about the control of ovulation cycle in mammals.

The analysis begins with the most simplified case of many growing follicles with same initial size and ageequivalent to only one growing follicle. The resulting equation is analytically integrable, and three possible behaviors are detected when the ageing parameter is not too large with respect to the initial size of the 



FIGURE 10 Solutions of follicles relative sizes for the anovulatory state with two initially "very old" follicles. Parameter values are $K=5.0, D=0.5, \mu=0.2, M_{1}=2.9$ and $M_{2}=3.9$, and initial age distribution as in Fig. 9. (a) There is not a significant qualitative difference from Fig. 5b, where the same follicles $x_{5}$ and $x_{6}$ are selected. However, follicles $x_{7}$ and $x_{8}$ atrophy slower than follicles $x_{7}$ and $x_{8}$ of Fig. 5b. (b) Corresponding numerical simulation of follicles relative oestradiol secretion to (a). The two selected follicles tend to the same relative maturity value $1 / 2$.

follicle. Whenever the ageing parameter is small enough so that it does not beat the selection process, the dynamics of a single follicle may present ovulatory, anovulatory and atretic behaviour for different values of the relevant parameters. This is nothing new to the results already obtained from Lacker's model.

For the ovulatory condition, however, a separatrix in the dynamics of Mariana and colleagues' model is found. Meaning that for a follicle size less than the threshold value, $X_{0}^{*}$ ovulation cannot occur, and the follicle atrophies. This may suggest that an initial sum of all growing follicles sizes has to exceed a minimum value so that ovulation can be triggered. It would be of great interest to biologically corroborate the fact that an ovary containing small follicles at the beginning of the menstrual cycle could be a possible cause of anovulation.

A new feature for the particular case of anovulation is reflected by this model. Namely, instead of having 
an arrested follicle with a fixed size, it eventually regresses due to its deteriorating capacity. Nevertheless, this regression occurs in a much more slower manner so that there is a visible difference between this follicle and an atretic one. This suggests that the pre-ovulatory follicle that did not manage to ovulate does eventually disappear, so that it may not remain inside the ovary for future cycles. However, since the model does not incorporate any time units, it is not possible to actually determine when the arrested follicle finally dies, nor the nature of this ageing process. Physiologically speaking, regression of stuck follicles could last for either months or years. Sometimes such cysts have to be surgically removed from a woman's ovary since their prolonged presence may produce some painful effects.

From the analysis of the simplified system, it is possible to estimate a threshold value for the age parameter. Such threshold depends on the follicles initial sizes and ages. Hence, a careful analysis is developed to show for both the ovulatory and anovulatory conditions when selection of pre-ovulatory follicles takes place. It is even possible to detect the relative values of the ageing parameter required for any follicular growth at the beginning of the cycle.

When further generalising the system by supposing that many follicles grow with different initial sizes, but still retaining the same initial age, the model can still be treated analytically. We prove that the model is still globally stable, and it still controls the number of pre-ovulatory selected follicles. The pre-destination of having the strictly largest follicles reaching a preovulatory stage is still maintained.

For the most general case of many interacting follicles with different sizes and ages, it is not possible to obtain a close expression for the non-linear stability analysis. The dynamics cannot be separated and therefore, it is not possible to find a gradient system for the interaction dynamics. However, a linear stability analysis is developed to show that, at least locally, the system for different follicles in age and size can still control the number of selected follicles. In other words, we prove that as long as the growing follicles begin with relatively similar initial ages and sizes, it is still possible to predict the number of follicles to reach pre-ovulatory maturity. Since the local analysis is around a trajectory rather than a fixed equilibrium point, the second order local analysis still needs to be developed.

Pre-destination of the system from the initial size of the follicles no longer holds. Some crossing between the follicles growth curves can result from this model. This is in better agreement with biological data since it has been shown that size is not the only factor determining selection. This fact indeed was already mentioned by Mariana and collaborators.

Numerical examples given in the present paper show that the model with an age decaying factor is not globally stable. When the system begins with, for instance, two "very old" follicles compared to the remaining ones, the number of expected ovulatory follicles is not maintained. This presents a tentative new insight into the selection of the control dynamics. This example in particular breaks the hierarchal structure for ovulation. For this case, from a certain number of ovulating follicles, there could be exactly the same number of stuck follicles. This suggests, that there are other local factors that affect follicular sensitivity to gonadotropins, and produce a polycystic ovary. Hence, the global instability presented by this model is not only suggesting but also agrees with alternative causes for PCO rather than those to do directly with follicle sensitivity to gonadotropins.

The biological interpretation of such an ageing factor is not specified. Although many biological entities decay at an exponential rate, the particular mechanisms through which follicles deteriorate are not defined through the ageing variable of this model. Moreover, the time scale of the ageing factor should be considered carefully. Therefore, this model supposes an atretic potential for all growing follicles. This type of atresia also, present in pre-ovulatory follicles, may interfere with the ovulation rate. Thus, whenever some of the largest follicles entering the follicular phase of the cycle are old enough, they will not be selected and may affect the response of the remaining large follicles to hormone stimulation. Therefore, no other pre-ovulatory follicles ovulate and remain within the ovary for an unspecified period of time. 
This is an alternative way of obtaining PCO in the human ovary. This particular model points towards the investigation of the local characteristics of the growing follicles that are not directly involved with their gonadotropin sensitivity. These characteristics can affect the global feedback mechanism and produce an undesirable PCO in women.

It would be of great interest to discern the origins of the ageing factor in order to provide a better biological understanding of the regulation of the ovulation number. An exponential decay for the ageing variable is plausible, yet by trying other types of decay rate, one could verify the robustness of the present model. However, the most important hypothesis this model actually suggests is that follicle sensitivity to gonadotropins can be strongly affected by this deteriorating factor. As a consequence, the system may no longer control the ovulation rate of preovulatory follicles.

I would like to thank Jaroslav Stark for his valuable contributions. I specially thank R. Carretero-González for all his support and aid towards the completion of this manuscript. Finally, I gratefully acknowledge the financial assistance of DGAPA-UNAM during my Ph.D. studies.

\section{References}

Akin, E. and Lacker, H.M. (1984) "Ovulation control: the right number or nothing", Journal of Mathematical Biology 20, $113-132$.
Bellman, R. (1960) Introduction to matrix analysis (McGraw-Hill, New York).

Chávez-Ross, A., Franks, S., Mason, H,D., Hardy, K. and Stark, J. (1997) "Modelling the control of ovulation and polycystic ovary syndrome", Journal of Mathematical Biology 36, 95-118.

Faddy, M.J. and Jones, M.C. (1988) "Fitting time-dependent multicompartment models: a case study", Biometrics 44, 587-593.

Gougeon, A. and Lefèvre, B. (1983) "Evolution of the diameters of the largest healthy and atretic follicles during the human menstrual cycle", Journal of Reproduction and Fertilization 69 , 497-502.

Hillier, S.G. (1994) "Current concepts of the roles of follicle stimulating hormone and luteinizing hormone in folliclegenesis", Human Reproduction 9(2), 188-191.

Hodgen, G.D. (1982) "The dominant ovarian follicle", Fertility and Sterility 38(3), 281-300.

Lacker, H.M. (1981) "Regulation of ovulation number in mammals. A follicle interaction law that controls maturation", Biophysical Journal 35, 433-454

Lacker, H.M. and Akin, E. (1988) "How do ovaries count?", Mathematical Biosciences 90, 305-332.

Lacker, H.M. and Percus, A. (1991) "How do ovarian follicles interact? A many-body problem with unusual symmetry and symmetry-breaking properties", Journal of Statistical Physics 63, $1133-1161$

Lacker, H.M., Beers, W., Mueli, L.E. and Akin, E. (1987) "A theory of follicle selection: I and II", Biology of Reproduction 37, $570-580$.

Ledger, W.L. and Baird, D.T. (1995) "Ovulation 3: Endocrinology of ovulation", In: Gvudzinskas, J.G. and Yovich, J.L., eds, From Gametes-The Oocyte (Cambridge University Press, UK), pp 193-209.

Mariana, J.C., Corpet, F. and Chevalet, C. (1994) "Lacker's model: control of follicular growth and ovulation in domestic species". Acta Biotheorica 42, 245-262.

Spears, N., Bruni, J.P. and Gosden, G. (1996) "The establishment of follicular dominance in co-cultured mouse ovarian follicles", Journal of Reproduction and Fertilization 106, 1-6. 


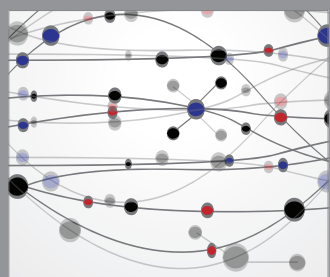

The Scientific World Journal
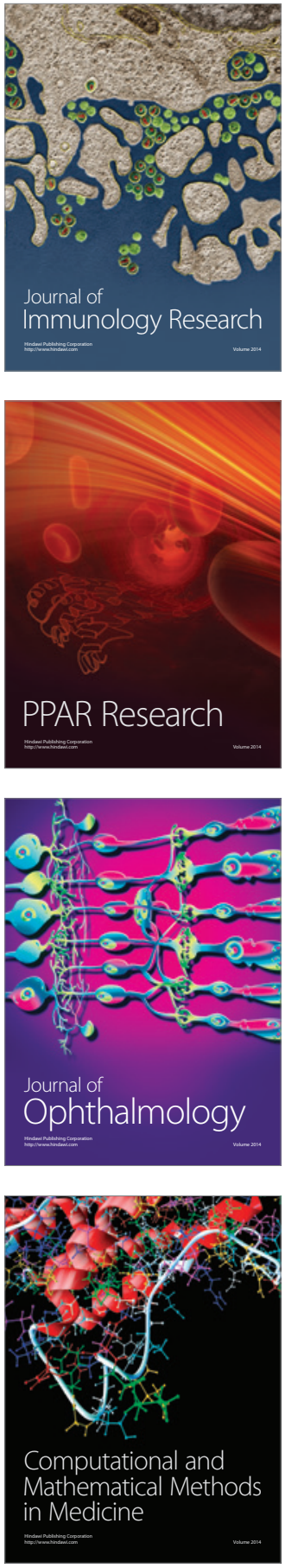

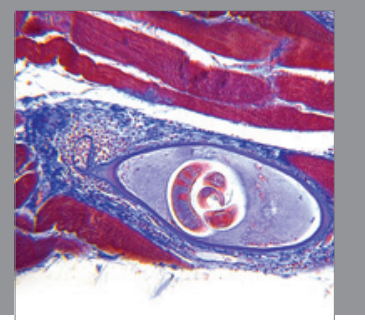

Gastroenterology

Research and Practice
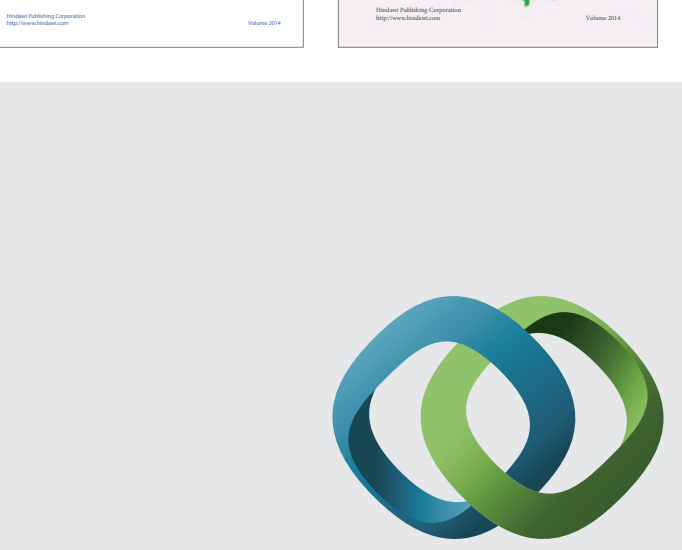

\section{Hindawi}

Submit your manuscripts at

http://www.hindawi.com
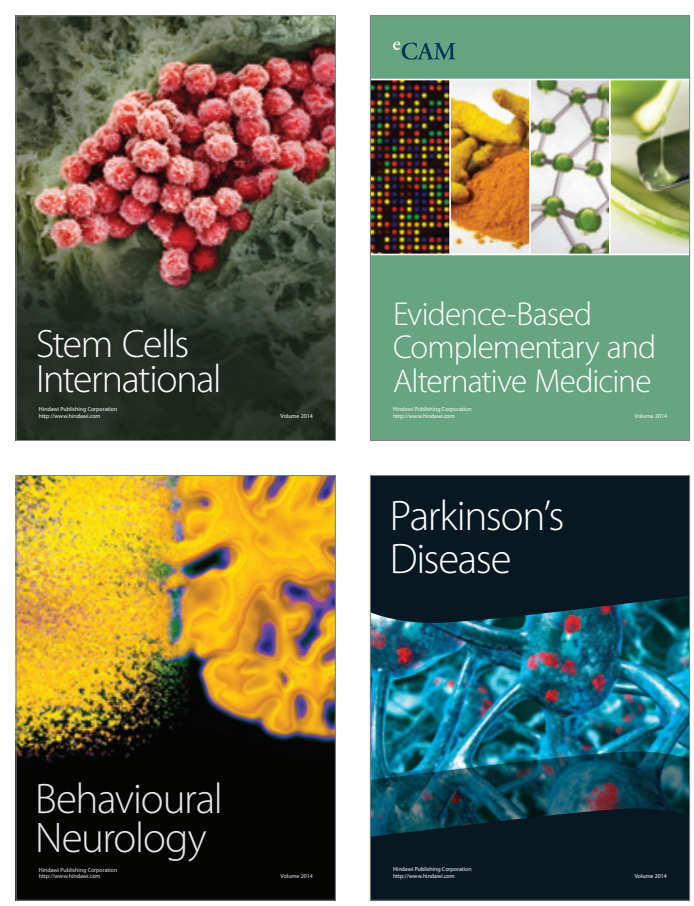

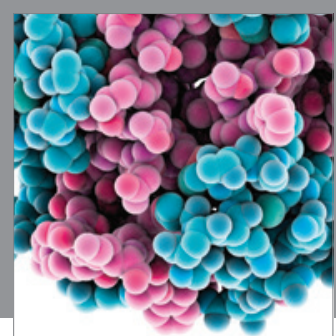

Journal of
Diabetes Research

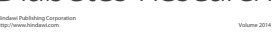

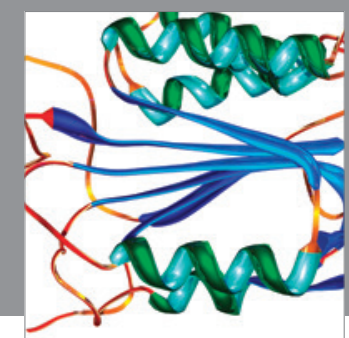

Disease Markers
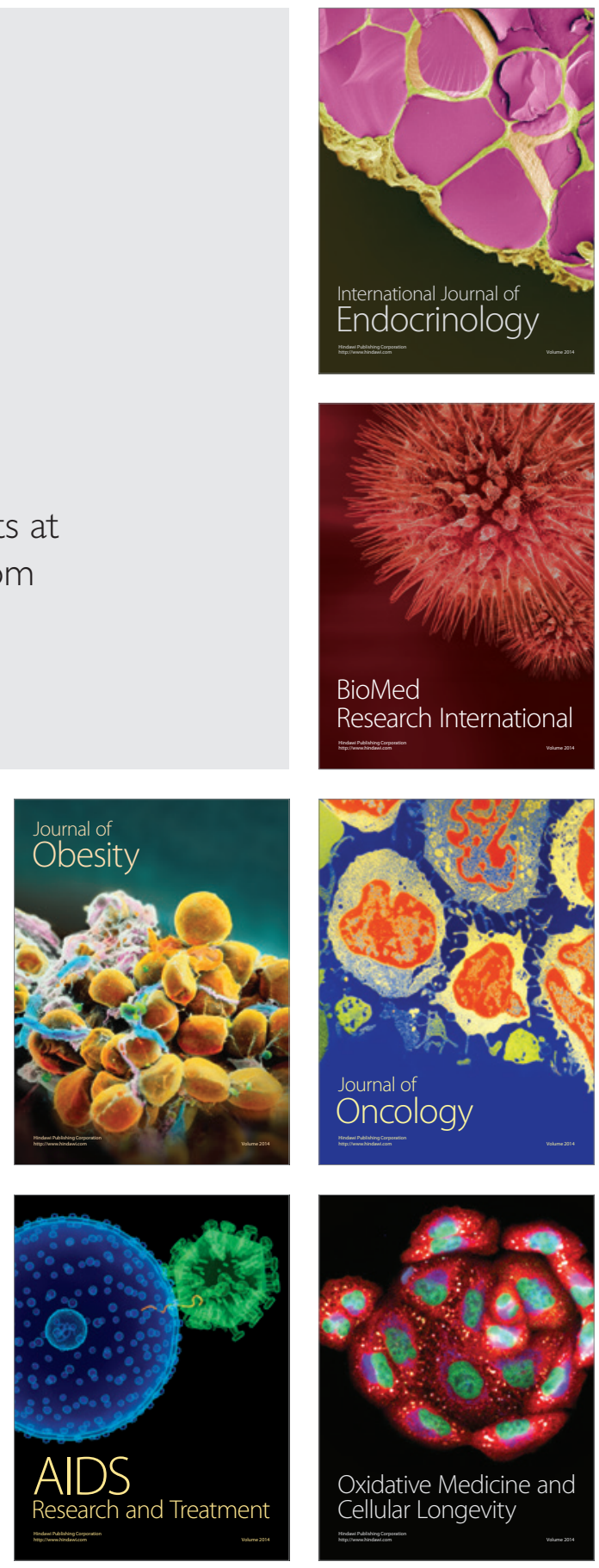